Bundesgesundheitsbl 2014 · 57:258-279

DOI 10.1007/s00103-013-1899-7

๑) Springer-Verlag Berlin Heidelberg 2014

Bekanntmachung des Umweltbundesamtes

\title{
Hygieneanforderungen an Bäder und deren Überwachung
}

\section{Empfehlung des Umweltbundesamtes (UBA) nach Anhörung der Schwimm- und Badebeckenwasserkommission des Bundesministeriums für Gesundheit (BMG) beim Umweltbundesamt}

\section{Präambel}

Im Infektionsschutzgesetz [1] wird in $\$ 37$ Absatz 2 die wichtigste Anforderung an die Beschaffenheit von Schwimm- und Badebeckenwasser gestellt:

"Schwimm- oder Badebeckenwasser in Gewerbebetrieben, öffentlichen Bädern sowie in sonstigen nicht ausschließlich privat genutzten Einrichtungen muss so beschaffen sein, dass durch seinen Gebrauch eine Schädigung der menschlichen Gesundheit, insbesondere durch Krankheitserreger, nicht zu besorgen ist."

Die Aufbereitung von Schwimm- und Badebeckenwasser muss so erfolgen, dass jederzeit in allen Beckenbereichen die Anforderungen des $₫ 37$ Absatz 2 IfSG erfüllt sind. Bei den Bädern, die normgerecht gebaut und betrieben werden, in denen die Wasseraufbereitung den allgemein anerkannten Regeln der Technik (a. a. R. d. T.) entspricht und bei denen insbesondere die Durchströmung, Aufbereitung und Betriebskontrolle normgerecht erfolgen (DIN 19643:2012-11 [2]), kann davon ausgegangen werden, dass eine hygienisch einwandfreie Wasserbeschaffenheit erzielt wird. Diese Anforde- rungen sind im Rahmen der allgemeinen Verkehrssicherungspflicht durch den Betreiber sicherzustellen und werden durch das Gesundheitsamt überwacht.

Die vorliegende Empfehlung legt insbesondere neben den mikrobiologischen und chemischen Anforderungen an die Qualität von Wasser in Schwimmund Badebecken, das in Gewerbebetrieben, öffentlichen Bädern sowie sonstigen nicht ausschließlich privat genutzten Einrichtungen zur Verfügung gestellt wird, auch Maßnahmen bei Nichteinhaltung der mikrobiologischen und chemischen Anforderungen fest. Sie formuliert hygienische Anforderungen an sonstige Einrichtungen in Bädern wie Barfußbereiche, Sitzflächen, raumlufttechnische Anlagen sowie an die Trinkwasser-Installation und gibt Hilfestellung, was beim Neubau eines Bades oder bei Änderungen an Schwimm- und Badebeckenanlagen beachtet werden muss.

Diese Empfehlung ersetzt $u$. a. wegen Neuerscheinung der DIN 19643:2012-11 die „Empfehlung des Umweltbundesamtes nach Anhörung der Schwimm- und Badebeckenwasserkommission des Bundesministeriums für Gesundheit beim Umweltbundesamt - Hygieneanforderungen an Bäder und deren Überwa- chung" [Bundesgesundheitsblatt-Gesundheitsforschung-Gesundheitsschutz 49, 9 (2006) 926-937].

\section{Begriffsbestimmungen}

Im Sinne dieser Empfehlung

- sind „Bäder“ sämtliche Einrichtungen in Gewerbebetrieben und öffentlichen sowie sonstigen nicht ausschließlich privat genutzten Einrichtungen, in denen Wasser in Schwimm- und Badebecken zur Verfügung gestellt wird,

- ist „Betreiber“ die natürliche oder juristische Person, die dafür verantwortlich ist, dass die gesetzlichen Bestimmungen und hygienischen Anforderungen in den ihrer Kontrolle unterstehenden Bädern erfüllt werden,

- sind "Schwimm- und Badebecken“ kontinuierlich durchströmte Wasserbecken, die dazu bestimmt sind, dass sich darin Menschen gleichzeitig oder nacheinander zum Schwimmen oder Baden aufhalten,

- sind „Schwimm- und Badebeckenanlagen" die Gesamtheit der Becken einschließlich der zugehörigen Anlagen zur Wasseraufbereitung, 
Tab. 1 Mikrobiologische Anforderungen an das Beckenwasser

\begin{tabular}{lll}
\hline Parameter & Parameterhöchstwert & Nachweisverfahren ${ }^{\mathrm{a}, \mathrm{b}}$ \\
\hline Pseudomonas aeruginosa & $0 / 100 \mathrm{ml}$ & DIN EN ISO 16266 \\
\hline Escherichia coli & $0 / 100 \mathrm{ml}$ & DIN EN ISO 9308-1 \\
\hline Legionella species & Siehe $\bullet$ Tab. 4 & ISO $11731^{\mathrm{d}}$ \\
& & DIN EN ISO 11731-2 \\
\hline Koloniezahl $\left(36^{\circ} \mathrm{C}\right)$ & $100 / \mathrm{ml}$ & DIN EN ISO 6222 \\
& & TrinkwV 2001 $^{\mathrm{c}}[3]$ \\
\hline
\end{tabular}

aEs dürfen die in der Tabelle genannten Nachweisverfahren oder gleichwertige Verfahren für Trink- und/oder Schwimm- und Badebeckenwasser nach DIN EN ISO 17994 eingesetzt werden. bDie Normen sind beim BeuthVerlag GmbH, 10772 Berlin, erhältlich. Im Beckenwasser von Warmsprudelbecken sowie Becken mit zusätzlichen aerosolbildenden Wasserkreisläufen und Beckenwassertemperaturen $\geq 23^{\circ} \mathrm{C}$. ${ }^{\mathrm{d} D i e ~ h i e r ~ a n g e g e b e n e n ~}$ 2 Nachweisverfahren sollen abdecken, dass bei der Untersuchung des Beckenwassers (und auch des Filtrates) sowohl ein Direktansatz ( $2 \times 0,5 \mathrm{ml}$ ausspateln nach ISO 11731) als auch ein Ansatz mit Membranfiltration (nach DIN EN ISO 11731-2) durchgeführt werden. Die Auswertung der Ansätze sollte nach der Empfehlung des UBA: http://www.umweltdaten.de/wasser/themen/trinkwasserkommission/internet-legionellen-empfehlung.pdf erfolgen. 'Bestimmung der Koloniezahl nach TrinkwV 2001, Anlage 5 Teil I, Punkt d), Unterpunkt bb).

- ist „Beckenwasser“ ein Synonym für den Begriff „Schwimm- und Badebeckenwasser",

- ist „Füllwasser“ das zur Erst- und Nachfüllung benutzte Wasser,

- ist „Filtrat“ das filtrierte Wasser vor Einmischung des Desinfektionsmittels,

- ist „Reinwasser“ Filtrat nach Einmischung des oxidierenden Desinfektionsmittels,

- ist „Gesundheitsamt“ die im Sinne des $\$ 2$ Nr. 14 des IfSG nach dem Landesrecht für die Durchführung dieses Gesetzes bestimmte und mit einem Amtsarzt besetzte Behörde,

- sind „Raumlufttechnische Anlagen“ die Gesamtheit der Anlagen u. a. gemäß DIN 1946 (s. auch Punkt 3.2) und

- ist „Trinkwasser-Installation“ die Gesamtheit der Rohrleitungen, Armaturen, Behälter und Geräte zwischen dem Punkt der Übergabe von Wasser aus einer Wasserversorgungsanlage und den Entnahmestellen einschließlich Duschen.

Keine Badebecken im Sinne dieser Empfehlung sind Wannen, deren Füllung nur je einer Person zur Verfügung gestellt wird und die nach jeder Benutzung entleert, gereinigt und desinfiziert sowie vor jeder Benutzung mit frischem Füllwasser versehen werden. Diesbezüglich wird auf die Veröffentlichung "Anforderungen an die Beschaffenheit des Wassers in Badeanlagen und Einrichtungen der Hydrotherapie“ im Bundesgesund- heitsblatt 31, 7 (1988) 253-254 hingewiesen. Für die Überwachung von Kleinbadeteichen im Sinne der Empfehlung des Umweltbundesamtes „Hygienische Anforderungen an Kleinbadeteiche (künstliche Schwimm- und Badeteichanlagen)“ [Bundesgesundheitsblatt-Gesundheitsforschung-Gesundheitsschutz 46, 6 (2003) 527-529] gilt diese Empfehlung nicht.

\section{Mikrobiologische und chemische Anforderungen an die Beschaffenheit von Schwimm- und Badebeckenwasser}

Um die Anforderungen an die Beschaffenheit von Schwimm- und Badebeckenwasser nach $₫ 37$ Absatz 2 IfSG zu erfüllen, sind die a. a. R. d. T. einzuhalten. Zudem ist ein optimales Zusammenwirken folgender Faktoren notwendig:

- Aufbereitung (Entfernung von Mikroorganismen und Belastungsstoffen),

- Desinfektion (Reduktion unerwünschter Mikroorganismen durch Abtötung oder Inaktivierung),

- Beckenhydraulik (optimale Verteilung des Desinfektionsmittels im gut durchströmten Becken und Austrag von Belastungsstoffen),

- im Wochendurchschnitt Zusatz von 301 Füllwasser pro Badegast (Verhinderung einer unerwünschten Anreicherung von Stoffen, die durch Aufbereitung nicht aus dem Wasser entfernt werden), wobei das Ergän- zungswasser nach der Spülung auf das Füllwasser angerechnet wird,

- Hinweise an die Badegäste über die unmittelbare vor dem Baden bestehende Notwendigkeit der Körperreinigung, um die Menge von organischem Material, die ins Beckenwasser eingebracht wird, zu reduzieren.

Die a. a. R. d. T. zum Erreichen der nachfolgenden mikrobiologischen, chemischen und physikalisch-chemischen Anforderungen an die Beckenwasserqualität sind in der Normenreihe DIN 19643:201211 „Aufbereitung von Schwimm- und Badebeckenwasser" festgelegt.

\subsection{Mikrobiologische Anforderungen}

Verschiedene Krankheitserreger können durch Wasser in Bädern auf Badegäste übertragen werden und z. B. Erkrankungen der Atemwege, des Magen- und Darmtraktes, der Leber, Augen, Ohren sowie der Haut hervorrufen. Der Nachweis, dass Beckenwasser keine fäkal-oral übertragbaren Krankheitserreger enthält, ist wegen der Vielzahl möglicher Krankheitserreger aus dieser Gruppe routinemäßig nicht durchzuführen. Deshalb wird die Konzentration von Indikatorbakterien, die ihrerseits auf das Vorhandensein von Krankheitserregern hinweisen können, bestimmt. Darüber hinaus werden 2 ausgewählte potenzielle Krankheitserreger mit Relevanz für das Beckenwasser (Pseudomonas aeruginosa und Legionella species) untersucht, da diese mittels Indikatorbakterien für fäkale Verunreinigungen nicht erfasst werden. Durch die Festlegung von Höchstwerten (- Tab. 1) soll ein Infektionsrisiko für den Badegast möglichst ausgeschlossen oder gering gehalten werden.

\section{Erläuterungen}

Bei dem Nachweisverfahren für Escherichia coli werden gleichzeitig weitere coliforme Bakterien mit erfasst, sofern sie in der Probe vorhanden sind. Dieser Nachweis sollte vom Labor als Nebenbefund angegeben werden. Er wurde in $\bullet$ Tab. 1 nicht berücksichtigt, weil keine zusätzlichen Erkenntnisse hinsichtlich einer fäkalen Kontamination gewonnen wer- 
Tab. 2 Parameterwerte für chemische und physikalisch-chemische Parameter im Beckenwasser

\begin{tabular}{|c|c|c|c|c|c|}
\hline $\begin{array}{l}\text { Lfd. } \\
\text { Nr. }\end{array}$ & Bezeichnung & $\begin{array}{l}\text { Unterer } \\
\text { Wert }\end{array}$ & $\begin{array}{l}\text { Oberer } \\
\text { Wert }\end{array}$ & $\begin{array}{l}\text { Anzuwendendes Re- } \\
\text { ferenzverfahren }^{\mathrm{a}}\end{array}$ & Bemerkungen \\
\hline 1 & $\begin{array}{l}\text { Freies Chlor }^{b} \\
\text { - Allgemein } \\
\text { - In Warmsprudelbecken }\end{array}$ & $\begin{array}{l}0,30 \mathrm{mg} / \mathrm{l} \\
0,70 \mathrm{mg} / \mathrm{l}\end{array}$ & $\begin{array}{l}0,60 \mathrm{mg} / \mathrm{l} \\
1,00 \mathrm{mg} / \mathrm{l}\end{array}$ & $\begin{array}{l}\text { DIN EN ISO 7393-1 } \\
\text { DIN EN ISO 7393-2 }\end{array}$ & $\begin{array}{l}\text { Die Bestimmung hat unmittelbar nach der Probennahme } \\
\text { vor Ort zu erfolgen. } \\
\text { Bei bromid- und jodidhaltigen Wässern wird freies Halo- } \\
\text { gen als Chlor bestimmt. } \\
\text { Die Konzentration an freiem Chlor kann vorübergehend } \\
\text { bis auf } 1,2 \mathrm{mg} / \mathrm{l} \text { erhöht werden, wenn die mikrobiologi- } \\
\text { schen Anforderungen auf anderem Wege nicht erfüllt } \\
\text { werden können. In diesen Fällen ist den Ursachen nach- } \\
\text { zugehen und für Abhilfe zu sorgen. } \\
\text { Bei bestimmten Verfahrenskombinationen kann ein } \\
\text { unterer Wert von } 0,2 \mathrm{mg} / \mathrm{l} \text { an Stelle von } 0,3 \mathrm{mg} / \mathrm{l} \text { ausrei- } \\
\text { chend sein, wenn der untere Wert für Parameter } 3 \text { nicht } \\
\text { unterschritten wird. }\end{array}$ \\
\hline 2 & $\begin{array}{l}\text { pH-Wert } \\
\text { Bei Flockung mit Aluminium oder } \\
\text { Aluminium-Eisen-Produkten } \\
\text { Bei Flockung mit Eisen-Produkten } \\
\text { a) Süßwasser } \\
\text { b) Meerwasser }\end{array}$ & $\begin{array}{l}6,5 \\
6,5\end{array}$ & $\begin{array}{l}7,5 \\
7,8\end{array}$ & DIN 38404-5 & $\begin{array}{l}\text { Die Bestimmung des } \mathrm{pH} \text {-Wertes erfolgt elektrometrisch } \\
\text { mit einer pH-Elektrode. }\end{array}$ \\
\hline 3 & $\begin{array}{l}\text { Redoxspannung gegen } \mathrm{Ag} / \mathrm{AgCl} \\
3,5 \mathrm{~m} \mathrm{KCl}\end{array}$ & $\begin{array}{l}750 \mathrm{mV}^{d} \\
770 \mathrm{mV}^{d} \\
700 \mathrm{mV}^{\mathrm{d}} \\
720 \mathrm{mV}^{\mathrm{d}}\end{array}$ & & DIN 384 & $\begin{array}{l}\text { Die Bestimmung hat in ortsfesten Mess- und Registrier- } \\
\text { geräten mit kontinuierlicher Messung zu erfolgen. Bei } \\
\text { Unterschreitung der Werte um }>50 \mathrm{mV} \text { sind die Funktion } \\
\text { und der Betrieb der Messeinrichtung und der Aufberei- } \\
\text { tungsanlage zu prüfen. Messwertangabe nur unter der } \\
\text { Bezeichnung der Bezugselektrode oder der Umrechnung. } \\
\text { Ablesung aus der betrieblichen Messwertanzeige. } \\
\text { Bei pH-Werten } \geq 6,5 \text { bis } \leq 7,3 \\
\text { Bei pH-Werten }>7,3 \text { bis } \leq 7,5 \\
\text { Bei pH-Werten } \geq 6,5 \text { bis } \leq 7,3 \\
\text { Bei pH-Werten }>7,3 \text { bis } \leq 7,8 \\
\text { Für Wasser mit einem Jodidanteil über } 0,5 \mathrm{mg} / \mathrm{l} \text { ist der } \\
\text { Wert für die ausreichende Redoxspannung experimentell } \\
\text { zu bestimmene. }\end{array}$ \\
\hline \multicolumn{6}{|c|}{$\begin{array}{l}\text { ¿Die Vorschriften für die angegebenen Referenzverfahren sind beim Beuth-Verlag GmbH, } 10772 \text { Berlin erhältlich und sind in den „Deutschen Einheitsverfahren zur Wasser-, } \\
\text { Abwasser- und Schlammuntersuchung“ (DEV), WILEY-VCH-Verlag GmbH und Co. KGaA, Weinheim, enthalten. 'bEine Justierung des Messwertgebers muss durchgeführt } \\
\text { werden, wenn es Abweichungen zwischen Anzeigegerät und der täglichen fotometrischen Kontrollmessung nach der DPD-Methode gibt. 'Die tägliche Funktionskontrolle } \\
\text { der fest installierten pH-Elektrode muss durch eine elektrometrische pH-Wert-Messung (Handmessgerät) vorgenommen werden. Abweichungen zwischen dem kontinuier- } \\
\text { lichen Anzeigegerät und dem Kontrollgerät dürfen nicht größer als } \pm 0,2 \text { pH-Einheiten sein. 'Für die Redoxspannung wird eine Messungenauigkeit von } \pm 40 \text { mV akzeptiert } \\
\text { (s. 2.3.1). eSeidel K.M., Lopez Pila J.M., Grohmann A.: Disinfection capability in water for swimming and bathing pools: A simple method for their evaluation in practice. Wat. } \\
\text { Sci. Technol. } 24 \text { (1991) 359-362. }\end{array}$} \\
\hline
\end{tabular}

den und coliforme Bakterien in der Regel kein erhöhtes gesundheitliches Risiko für den Badegast darstellen. Der Nachweis von E. coli als Fäkalindikatorbakterium ist ausreichend als Hinweis auf eine fäkale Verunreinigung und kann auf das Vorhandensein von Durchfallerregern hinweisen. In seltenen Fällen kann E. coli selbst Infektionen verursachen. Das alleinige Vorhandensein von coliformen Bakterien ohne E. coli weist auf eine nicht ausreichende Funktion der Desinfektion oder eine Biofilmbildung im Wasserkreislauf (meist Filter) hin.

Pseudomonas aeruginosa ist ein Erreger Schwimmbad-assoziierter Infektionskrankheiten. Vor allem Infektionen der
Haut und des Außenohrs können durch ihn hervorgerufen werden. Besonders anfällig sind hierbei Personen mit einer vorgeschädigten Haut oder mit kleinen Wunden. Pseudomonas aeruginosa ist eine Bakterienart, deren Vertreter sich durch die Ausbildung einer extrazellulären Schleimschicht vor widrigen Einflüssen, wie z. B. Desinfektionsmitteln, schützen können. Ihr Vorhandensein weist auf mögliche Mängel bei dem Filterbetrieb, auf eine unzureichende Beckenwasserdesinfektion und/oder auf eine mangelhafte Beckendurchströmung, auf Mängel bei der Reinigung und Desinfektion der Becken sowie bei der Materialauswahl hin.
Legionellen sind Bakterien, bei denen es in einem Temperaturbereich zwischen $23^{\circ} \mathrm{C}$ und $50^{\circ} \mathrm{C} \mathrm{zu}$ einer hygienisch relevanten Vermehrung kommen kann. Sie können in geringer Zahl über das Füllwasser eingetragen werden und sich bei nicht ausreichender Desinfektion und Spülung vor allem in den Filtern vermehren. Als Hauptinfektionsweg gilt das Einatmen Legionellen-haltiger Aerosole, aber auch die Mikroaspiration Legionellen-haltigen Wassers kann zu Infektionen führen. Als Krankheitsbilder treten das Grippe-ähnliche Pontiac-Fieber oder atypische Lungenentzündungen mit $\mathrm{z}$. T. schwerem Krankheitsverlauf auf. Indikatorbakterien für das Vorhandensein von 
Tab. 3 Parameterhöchstwerte für Desinfektionsnebenprodukte und Arsen im Beckenwasser

\begin{tabular}{|c|c|c|c|c|}
\hline $\begin{array}{l}\text { Lfd. } \\
\text { Nr. }\end{array}$ & Bezeichnung & $\begin{array}{l}\text { Oberer } \\
\text { Wert }\end{array}$ & $\begin{array}{l}\text { Anzuwendendes } \\
\text { Referenzverfahren }^{\mathrm{a}}\end{array}$ & Bemerkungen \\
\hline 1 & $\begin{array}{l}\text { Gebundenes } \\
\text { Chlor }{ }^{\mathrm{b}, \mathrm{d}, \mathrm{e}}\end{array}$ & $0,20 \mathrm{mg} / \mathrm{l}$ & $\begin{array}{l}\text { DIN EN ISO 7393-1 } \\
\text { DIN EN ISO 7393-2 }\end{array}$ & $\begin{array}{l}\text { Die Bestimmung hat unmittelbar } \\
\text { nach der Probennahme vor Ort zu } \\
\text { erfolgen. Der Gehalt an gebunde- } \\
\text { nem Chlor wird berechnet aus der } \\
\text { Differenz zwischen dem Gehalt an } \\
\text { Gesamtchlor und dem Gehalt an } \\
\text { freiem Chlor. } \\
\text { Bei bromid- oder jodidhaltigen } \\
\text { Wässern wird gebundenes Halogen } \\
\text { als Chlor bestimmt. }\end{array}$ \\
\hline 2 & $\begin{array}{l}\text { Trihalogenme- } \\
\text { thane THM }{ }^{b, c, d, e}\end{array}$ & $0,020 \mathrm{mg} / \mathrm{l}$ & $\begin{array}{l}\text { DIN 38407-30 } \\
\text { DIN EN ISO } 15680 \\
\text { DIN EN ISO 10301: } \\
1997 \text { (Verfahren 2) }\end{array}$ & $\begin{array}{l}\text { Berechnet als Chloroform }\left(\mathrm{CHCl}_{3}\right) \\
\mathrm{THM}=\mathrm{A}+0,728 \times \mathrm{B}+0,574 \times \mathrm{C} \\
+0,472 \times \mathrm{D} \\
\left(\mathrm{A}=\mathrm{mg} / \mathrm{l} \mathrm{CHCl} \mathrm{CH}_{3} ; \mathrm{B}=\mathrm{mg} / \mathrm{lCHBrCl}_{2} ;\right. \\
\left.\mathrm{C}=\mathrm{mg} / \mathrm{l} \mathrm{CH} \mathrm{Br} 2 \mathrm{Cl} ; \mathrm{D}=\mathrm{mg} / \mathrm{lCHBr}_{3}\right)\end{array}$ \\
\hline 3 & $\begin{array}{l}\text { Summe Chlorit } \\
+ \text { Chlorat }^{f}\end{array}$ & $30 \mathrm{mg} / \mathrm{l}$ & DIN EN ISO 10304-4 & \\
\hline 4 & Bromat & $2,0 \mathrm{mg} / \mathrm{l}$ & $\begin{array}{l}\text { DIN EN ISO } 15061 \\
\text { ISO/DIS } 11206\end{array}$ & \\
\hline 5 & Arsen & $0,2 \mathrm{mg} / \mathrm{l}$ & $\begin{array}{l}\text { DIN 38405-35 } \\
\text { DIN EN ISO } 11969 \\
\text { DIN EN ISO } 11885 \\
\text { DIN EN ISO } 17294-2\end{array}$ & $\begin{array}{l}\text { Die Bestimmung ist nur notwendig } \\
\text { bei Verwendung von Füllwasser } \\
\text { mit erhöhten Arsengehalten (z. B. } \\
\text { Thermalwasser). }\end{array}$ \\
\hline
\end{tabular}

aDie Vorschriften für die angegebenen Referenzverfahren sind beim Beuth-Verlag GmbH, 10772 Berlin erhältlich und sind in den „Deutschen Einheitsverfahren zur Wasser-, Abwasser- und Schlammuntersuchung" (DEV), Wiley-Verlag GmbH und Co. KgaA, Weinheim, enthalten ' $\mathrm{G}$ Gelegentliche Überschreitungen des oberen Wertes um bis zu 20\% sind in der Bewertung tolerierbar. 'Bei Freibädern während höherer Chlorung zur Einhaltung der mikrobiologischen Anforderungen dürfen höhere Werte auftreten. ${ }^{\mathrm{d}}$ Gilt nicht für Kaltwassertauchbecken $\leq 2 \mathrm{~m}^{3}$ wenn sie kontinuierlich mit gechlortem Füllwasser durchströmt werden, das Wasser über eine Überlaufrinne abfließt, Verdrängungswasser so schnell wie möglich ergänzt wird und die Becken täglich entleert, gereinigt und neu gefüllt werden. Das Füllwasser ist so zu chloren, dass das Beckenwasser 0,3 bis 0,6 mg/l freies Chlor enthält. eBei Kaltwassertauchbecken, bei denen sichergestellt ist, dass die Wassertemperatur $15^{\circ} \mathrm{C}$ nicht überschreitet, braucht dieser Wert nicht beachtet zu werden. . Bestehende Anlagen, die diese Anforderungen nicht erfüllen, sollten bis November 2017 in die Lage versetzt werden, diese Werte zu unterschreiten.

Legionellen gibt es nicht. Deshalb muss in Becken, die eine Wassertemperatur von über $23^{\circ} \mathrm{C}$ aufweisen und in denen Aerosole entstehen können, zusätzlich auf Legionella species untersucht werden. Dies gilt insbesondere für Warmsprudelbecken, die in der Vergangenheit gelegentlich Ursache für Legionellen-bedingte Atemwegsinfektionen waren.

Die Koloniezahl ist ein Indikatorparameter. Die Bestimmung der Koloniezahl gibt Auskunft über den allgemeinen hygienischen Status der Schwimm- und Badebeckenanlage und die Qualität der Aufbereitung.

Beim Auftreten von gehäuften Infektionsfällen ist das Beckenwasser gezielt auf die infrage kommenden Krankheitserreger zu untersuchen. Neben $P$. aeruginosa und Legionellen wurden Cryptosporidien, Viren, speziell Noroviren, atypische Mykobakterien und enterohä- die in $\bullet$ Tab. 2 zusammengestellten chemischen und physikalisch-chemischen Parameter sind einzuhalten. Hierbei handelt es sich um Parameter der Aufbereitung einschließlich der Desinfektion.

\section{Erläuterungen}

Jede mikrobiologische Untersuchung erlaubt stets nur für den Zeitpunkt der Probennahme eine Aussage über den seuchenhygienischen Zustand des Schwimm- und Badebeckenwassers. Da sich die Wasserbeschaffenheit schnell ändern kann und es für die Überwachung wichtig ist $\mathrm{zu}$ wissen, ob die Wasserbeschaffenheit während der Zeit zwischen 2 Untersuchungsterminen einwandfrei war, ist es erforderlich, außer den mikrobiologischen Parametern die chemischen Hygiene-Hilfsparameter freies Chlor, $\mathrm{pH}$-Wert und Redoxspannung in größerer zeitlicher Dichte zu messen. Die Messung dieser Parameter sollte automatisch und kontinuierlich erfolgen, um eine lückenlose Beurteilung der seuchenhygienischen Verhältnisse im Beckenwasser ohne zeitliche Verzögerung zu ermöglichen. Bei Verwendung von Chlor oder den in der DIN 19643:2012-11 aufgeführten Chlorverbindungen besteht ein direkter Zusammenhang zwischen der Desinfektionswirkung des freien Chlors und der Redoxspannung. Die Redoxspannung im Schwimm- und Badebeckenwasser ist abhängig von der Konzentration des freien Chlors, dem $\mathrm{pH}$-Wert sowie von den Belastungsstoffen im Beckenwasser. Sie dient als Indikator für die Desinfektionskapazität des gechlorten Beckenwassers und ermöglicht indirekt einen Rückschluss auf die kontinuierliche Einhaltung der mikrobiologischen Anforderungen. So ist z. B. Beckenwasser (Süßwasserbad) mit einer Redoxspannung von $\geq+750 \mathrm{mV}$ bei $\mathrm{pH}$-Werten $\geq 6,5$ bis $\leq 7,3$, gemessen gegen die Referenzelektrode Silber/Silberchlorid, als ausreichend desinfiziert zu beurteilen. Ein Absinken der Redoxspannung bei gleichzeitiger Einhaltung des freien Chlorwertes weist auf eine Fehlfunktion der Aufbereitung oder eine Überlastung des Beckens hin.

Freies Chlor liegt im Beckenwasser infolge seiner Disproportionierung als hypochlorige Säure (frühere Bezeich- 
nung: unterchlorige Säure) $(\mathrm{HOCl})$ und Hypochlorit-Anion $\left(\mathrm{OCl}^{-}\right)$vor. Die Verteilung zwischen den beiden Verbindungen ist $\mathrm{pH}$-Wert abhängig, wobei die Desinfektionswirkung im Wesentlichen durch die hypochlorige Säure bewirkt wird. Mit steigendem $\mathrm{pH}$-Wert nimmt die desinfizierende Wirkung des Systems hypochlorige Säure/Hypochlorit ab, da sich das Gleichgewicht zuungunsten der Säure verschiebt. Bei gleichem Gehalt an freiem Chlor ist deshalb die Desinfektionswirkung der Chlorung bei $25^{\circ} \mathrm{C}$ bei pH 6,5 um den Faktor 1,7 größer als bei pH 7,5 und um den Faktor 9,3 größer als bei pH 8,5. Da der pH-Wert auch von wesentlicher Bedeutung für die Flockung, für die Korrosionseigenschaften des Beckenwassers und für dessen physiologische Hautverträglichkeit ist, sollte er neben der Redoxspannung und dem freien Chlor kontinuierlich gemessen und aufgezeichnet sowie hinsichtlich der Einhaltung der Sollwerte kontrolliert werden.

\subsubsection{Weitere chemische und physi- kalisch-chemische Anforderungen}

Die unteren bzw. oberen Parameterwerte für Färbung, Trübung, Aluminium, Eisen, Säurekapazität, Nitrat und Oxidierbarkeit nach Tabelle 2 der DIN 19643: 2012 11 sind einzuhalten.

\subsubsection{Desinfektionsneben- produkte und Arsen}

Bei der Desinfektion von Schwimmund Badebeckenwasser entstehen diverse gesundheitlich bedenkliche Desinfektionsnebenprodukte (DNP), die in ihrer Konzentration minimiert werden müssen. Aus der Gruppe der Desinfektionsnebenprodukte werden stellvertretend die nachfolgend aufgeführten Stoffe gemessen und die Einhaltung toxikologisch vertretbarer und technisch einhaltbarer Konzentrationen überwacht.

\section{Erläuterungen}

Die Trihalogenmethane (THM), Trichlormethan (Chloroform), Bromdichlormethan, Dibromchlormethan und Tribrommethan (Bromoform) bilden sich bei der Reaktion des freien Chlors mit im Beckenwasser vorhandenen Vorläuferverbindungen (organische Stof- fe, die von Badegästen im Wasser abgegeben werden; Huminstoffe im Füllwasser). Die THM sind leichtflüchtig und gasen deshalb aus dem Wasser aus. In Hallenbädern können dadurch eine Anreicherung der THM in der Hallenbadluft und eine Belastung der Badenden und des Schwimmbadpersonals durch THM über die Atmung auftreten. Die THMBelastung über die Atmung in Freibädern ist dagegen gering, weil es in diesen Bädern durch ständigen Luftwechsel nicht $\mathrm{zu}$ einer Anreicherung in der Atemluft kommen kann. Kritisches Zielorgan für die toxische und karzinogene Wirkung der THM sind Leber und Niere. Aufgrund dieser möglichen Wirkung wird die THM-Konzentration im Beckenwasser mit einem oberen Wert (Vorsorgewert) versehen (• Tab. 3). Der THMGehalt wird vereinbarungsgemäß auf die Verbindung mit dem geringsten Molekulargewicht (Chloroform) umgerechnet.

Unter dem Summenparameter ,gebundenes Chlor" werden weitere Nebenprodukte der Chlorung zusammengefasst. Es handelt sich dabei um Derivate des Ammoniaks, bei denen 1, 2 oder 3 Wasserstoffatome durch Chloratome ersetzt wurden [Monochloramin $\mathrm{NH}_{2} \mathrm{Cl}$, Dichloramin $\mathrm{NHCl}_{2}$, Stickstofftrichlorid $\mathrm{NCl}_{3}$ (frühere Bezeichnung: Trichloramin)], und um chlorierte Derivate von organischen Stickstoffverbindungen. Vorläuferverbindung für die 3 anorganischen Chloramine ist vor allem Harnstoff, der von den Badegästen in das Beckenwasser eingebracht wird (Ausspülen aus der Hornschicht der Haut, Eintrag von Urin und Schweiß).

Harnstoff ist eine wichtige Feuchthaltesubstanz der Haut. Die Hornschicht (Stratum corneum) als äußere Schicht der Oberhaut enthält ca. $8 \mu \mathrm{g}$ Harnstoff pro $\mathrm{cm}^{2}$ [4]. Geht man davon aus, dass die Haut eines Erwachsenen ca. $2 \mathrm{~m}^{2}$ Fläche einnimmt und der Harnstoff der Haut als wichtigste Vorläufersubstanz für die Bildung von gebundenem Chlor vollständig vom Beckenwasser beim Schwimmen oder Baden ausgespült wird, dann werden pro Badegast durchschnittlich 0,16 g Harnstoff über die Haut ins Wasser eingebracht. Der Harnstoff lässt sich durch gründliches Duschen fast vollständig aus der Haut entfernen. Das Beispiel verdeut- licht den hohen Stellenwert einer gründlichen Körperreinigung zur Verringerung der unerwünschten Chloraminbildung im Beckenwasser. Insbesondere das zum gebundenen Chlor zählende Stickstofftrichlorid ist ein Reizstoff für Augen, Nase, Rachen und Bronchien. Es besitzt eine Geruchs- und Geschmacksschwelle von $0,02 \mathrm{mg} / \mathrm{l}$, gast wie die THM aus dem Beckenwasser aus und sorgt für den sog. Hallenbadgeruch. Da das gebundene Chlor die Beckenwasserqualität erheblich beeinträchtigt, muss es als belastendes Nebenprodukt der Chlorung auf die technisch unvermeidbare Konzentration begrenzt werden.

In die DIN 19643:2012-11 wurden als neue chemische Parameter für das Beckenwasser die anorganischen Desinfektionsnebenprodukte Chlorit, Chlorat und Bromat aufgenommen und mit einem oberen Wert begrenzt. Das freie Chlor im Beckenwasser setzt sich aus hypochloriger Säure $(\mathrm{HOCl})$ und Hypochlorit-Ionen $\left(\mathrm{OCl}^{-}\right)$zusammen (s. Pkt. 2.2.1). In wässriger Lösung zerfallen die Hypochlorit-Ionen in einer zweistufigen Reaktion zu Chlorat $\left(\mathrm{ClO}_{3}{ }^{-}\right)$. In der ersten Stufe wird Chlorit $\left(\mathrm{ClO}_{2}^{-}\right)$gebildet, das in Gegenwart von weiterem Hypochlorit sofort zu Chlorat weiter reagiert. Deshalb ist in einem Beckenwasser, das ausreichend freies Chlor (• Tab. 2) enthält, kein Chlorit vorhanden. Die Disproportionierung (Zerfall) von Hypochloriten zu Chlorit und Chlorat wird begünstigt durch hohe Hypochlorit-Konzentrationen (z. B. konzentrierte Natriumhypochlorit-Lösung), Wärme, UV-Strahlung (Sonnenlicht), $\mathrm{pH}$-Werte $<10,5$ und Katalysatoren (z. B. Schwermetallionen). So kann es insbesondere durch den Zerfall von Hypochlorit im Beckenwasser von Freibädern an heißen Sommertagen (starke UV-Strahlung, höhere Temperaturen) zur Bildung von Chlorat kommen. Eine wesentliche Chlorat-Quelle stellt gealterte Natriumhypochlorit-Lösung („Chlorbleichlauge“) dar. Höhere Temperaturen, Lichteinstrahlung und lange Lagerzeiten führen zum beschleunigten $\mathrm{Ab}$ bau des freien Chlors unter Bildung von Chlorat. Beim Einsatz von Chlorgas zur Desinfektion von Beckenwasser in Hallenbädern ist die Gefahr der Bildung von Chlorat nicht gegeben. Beim Einsatz von 
Calciumhypochlorit (Tabletten, Granulat) findet eine Bildung von Chlorat nur statt, wenn vor Ort hergestellte Lösungen unter ungünstigen Bedingungen gelagert werden.

In Freibädern kann es aufgrund der Sonneneinstrahlung aber generell zur Chloratbildung kommen, unabhängig von der Art des eingesetzten Desinfektionsmittels.

Der toxikologische Hintergrund für eine Begrenzung der Chlorit/ChloratKonzentration im Beckenwasser besteht vorrangig in der Schädigung der roten Blutkörperchen (Methämoglobin-bildende Stoffe) und in der nierenschädigenden Wirkung.

Chlorat lässt sich nicht durch die Wasseraufbereitung aus dem Beckenwasser entfernen. Die Chloratkonzentration kann deshalb nur durch die Verminderung des Chlorateintrags über chlorathaltige Desinfektionslösungen und durch Verdünnung mit Füllwasser in Grenzen gehalten werden.

Ausführlich wird die Chlorit- und Chloratproblematik im Beckenwasser in [5] beschrieben.

Das anorganische Desinfektionsnebenprodukt Bromat $\left(\mathrm{BrO}_{3}{ }^{-}\right)$wurde in die DIN 19643:2012-11 als chemischer Qualitätsparameter für das Beckenwasser neu aufgenommen und ein oberer Wert (Maximalwert) von $2 \mathrm{mg} / \mathrm{l}$ für diesen Parameter festgelegt. Dieser toxikologisch begründete Wert wurde vom UBA abgeleitet. Bromat ist ein nicht-gentoxisches Karzinogen mit relativ niedrigem karzinogenem Potenzial, Zielorgan ist die Niere.

Bei nicht-gentoxischen Substanzen wird die Existenz eines Schwellenwertes angenommen, sodass für diese Stoffe duldbare Aufnahmemengen abgeleitet werden können, bei deren Einhaltung nicht mit einem gesundheitsschädlichen Effekt gerechnet werden muss.

Bromat kann auf 2 unterschiedlichen Wegen in das Beckenwasser gelangen. So entsteht durch Oxidation von Bromid mit Ozon bei der Beckenwasseraufbereitung Bromat (Verfahrenskombination nach DIN 19643-3: 2012-11). Die Quelle für das Bromid im Beckenwasser ist das Füllwasser (Meer-, Thermal-, Mineral-, Heilwasser und Sole). Die Konzentration des ge- bildeten Bromats hängt vor allem von folgenden Parametern ab: Bromidkonzentration im Beckenwasser, Ozondosis, Reaktionszeit mit Ozon, pH-Wert. Während die Bromidkonzentration durch das Füllwasser vorgegeben ist, greifen Bromat-limitierende Maßnahmen beim pH-Wert, bei der Ozondosis und der Reaktionszeit mit Ozon. Durch Herabsetzung des pHWertes auf $\mathrm{pH}<7,0$ wird bei gleichbleibender Ozonexposition die Bromatbildung sehr effektiv eingeschränkt. Darüber hinaus können folgende Möglichkeiten der Bromatminimierung genutzt werden: Nach der Norm DIN 19643-3: 201211 darf die geforderte Ozonkonzentration von $0,3 \mathrm{mg} / \mathrm{l}$ auf eine Mindestkonzentration von $0,1 \mathrm{mg} / \mathrm{l}$ Ozon reduziert werden, wenn die Ozonzugabe in Abhängigkeit einer kontinuierlichen Bestimmung des gebundenen Chlors des Beckenwassers geregelt wird.

Kürzere Reaktionszeiten des gelösten Ozons unter 3 min sind dann zulässig, wenn damit die Anforderungen nach DIN 19643-1: 2012-11, Tabellen 1 und 2, erfüllt werden.

Eine Reduzierung der Ozonkonzentration und der Reaktionszeit des Ozons ist für Therapiebecken nicht erlaubt.

Bromat kann aber auch als Verunreinigung der zur Desinfektion eingesetzten Natriumhypochlorit-Lösung ins Beckenwasser eingebracht werden. Ausschlaggebend für den Bromatgehalt einer handelsüblichen oder auch in einer Elektrolyseanlage vor Ort hergestellten Natriumhypochlorit-Lösung ist der Bromidgehalt des eingesetzten Elektrolysesalzes. Je geringer der Bromidgehalt des zur Elektrolyse verwendeten Salzes ist, desto geringer wird der Bromatgehalt in der hergestellten Natriumhypochlorit-Lösung sein.

In der DIN EN 15077: 2013-08 „Produkte zur Aufbereitung von Schwimmund Badebeckenwasser - Natriumhypochlorit" sind die Zusammensetzung und die Reinheitskriterien für handelsübliche Natriumhypochlorit-Lösungen festgelegt. Nach dieser Norm müssen konzentrierte Lösungen mindestens $12 \%$ Aktivchlor (wirksames Chlor) enthalten. Der Gehalt an Natriumchlorat $\left(\mathrm{NaClO}_{3}\right)$ als Nebenprodukt des Herstellungsprozesses darf in diesen Lösungen einen Massenanteil von 5,4\% an Aktivchlor zum
Zeitpunkt der Lieferung durch den Hersteller nicht überschreiten. Die Produktkennzeichnung muss folgende Angabe enthalten „Dieses Produkt entspricht EN 15077“. Wenn vom Hersteller nicht anders angegeben, sollte die Chlorbleichlauge möglichst nicht länger als 3 Monate im Bad gelagert werden. Die Lagerung sollte möglichst unter $20^{\circ} \mathrm{C}$ im Dunkeln erfolgen. Erfolgt die Lagerung und Dosierung der Chlorbleichlauge aus einem Vorratstank, so ist darauf zu achten, dass dieser regelmäßig, aber mindestens einmal im Jahr gereinigt wird. Vom Hersteller von Geräten zur elektrolytischen Chlorerzeugung im Bad ist anzugeben, wie hoch der Gehalt an aktivem Chlor, Chlorat und Bromat bei ordnungsgemäßem Betrieb liegt.

In die Norm DIN 19643-1: 2012-11 wurde auch Arsen als chemischer Beckenwasserqualitätsparameter neu aufgenommen. Eine Arsenkonzentration von $0,2 \mathrm{mg} / \mathrm{l}$ darf im Beckenwasser nicht überschritten werden. Der Parameter ist nur dann im Beckenwasser zu untersuchen, wenn arsenhaltige Füllwässer (z. B. Heilwässer) zum Einsatz kommen. Epidemiologische Studien belegen, dass Arsen ein systemisch wirkendes Karzinogen für die Organe Haut, Harnblase, Leber, Lunge und eine Reihe weiterer Organe darstellt.

Neben der Einhaltung der in den - Tab. 2 und 3 aufgeführten chemischen Parameter sind weitere chemische Parameter nach DIN 19643-1:2012-11 in die Betriebskontrolle der Wasserbeschaffenheit mit einzubeziehen, damit gewährleistet werden kann, dass die Wasseraufbereitung in einem optimalen Zustand betrieben wird (Abschn. 2.3.1).

\subsubsection{Vermeiden von Algenaufwuchs}

Bei der Flockung findet eine Phosphateliminierung statt. Dadurch wird den Algen ein lebenswichtiger Nährstoff entzogen oder so stark minimiert, dass ein Algenwachstum verhindert wird. Der Einsatz von Algiziden zur Bekämpfung von Algen ist unerwünscht und entbehrlich. Die übliche Chlorung des Beckenwassers im Zusammenwirken mit der Beckenhydraulik ist dann ausreichend, um einen algistatischen Zustand im Becken- 
wasser aufrechtzuerhalten. Der Einsatz von Algiziden zur Bekämpfung von $\mathrm{Al}$ genbewuchs ist dadurch überflüssig. In diesen Produkten sind als wirksame Komponenten polyquaternäre Ammoniumverbindungen enthalten. Sie tragen zwar nicht zur Bildung von gebundenem Chlor, Stickstofftrichlorid oder THM bei, verursachen aber eine erhöhte Chlorzehrung und vermehrte Bildung von adsorbierbaren organischen Halogenverbindungen (AOX). Einige dieser Produkte neigen darüber hinaus zur Schaumbildung im Beckenwasser. Treten schwarze oder grüne Flecken (Algenbewuchs) an den Beckenwänden auf, sind die Flecken mechanisch zu entfernen.

\section{3 Überwachung}

Der Betreiber eines Bades hat die Verkehrssicherheit wie u. a. die zuverlässige Abwesenheit von Krankheitserregern $^{1}$ und toxikologisch relevanter chemischer Stoffe zu gewährleisten. Dies wird grundsätzlich als gegeben angesehen, wenn er die a. a. R. d. T. (DIN, Deutsche Gesellschaft für das Badewesen, Regelwerke von Fachverbänden) einhält. Er ist dementsprechend zur Eigenüberwachung verpflichtet. Aufgrund ihrer hohen Bedeutung werden die Anforderungen an die Hygiene auch vom Gesundheitsamt überwacht. Hierbei obliegt diesem sowohl die Kontrolle der Einhaltung der Betreiberverpflichtungen als auch die Überwachung von Beckenwasser einschließlich der Wasseraufbereitungsanlagen nach $₫ 37$ Absatz 3 IfSG hinsichtlich der Anforderungen der Hygiene. Für die Durchführung der Überwachung gilt $\$ 16$ Absatz 2 IfSG. Gemäß $\$ 39$ Absatz 2 IfSG ordnet das Gesundheitsamt bzw. die zuständige Behörde die notwendigen Maßnahmen zur Einhaltung der Hygieneanforderungen des $₫ 37$ Absatz 2 IfSG und zur Verhinderung von Gesundheitsgefahren an einschließlich der Eigenüberwachungen, soweit diese nicht bereits durchgeführt werden.

Sofern die Landesgesetze für den Öffentlichen Gesundheitsdienst nichts anderes vorsehen, greift das Gesundheits-

\footnotetext{
1 In gesundheitsgefährdenden Konzen-
} trationen. amt bei der Überwachung der Anforderungen der Hygiene an die sonstigen Einrichtungen in den Bädern auf die allgemeinen Anforderungen des $\$ 16$ Absatz 1 IfSG zurück. Hierzu gehört im begründeten Einzelfall auch die Möglichkeit, den Betreiber über das Maß seiner Eigenüberwachung gemäß 2.3.1 hinaus zu zusätzlichen Untersuchungen zu verpflichten.

Das gesamte System der Trinkwasser-Installation unterliegt den Anforderungen und dem Regelungsbereich der TrinkwV 2001 und damit ebenfalls der Überwachung durch das Gesundheitsamt.

Die Überwachung durch das Gesundheitsamt kann nur dann erfolgreich sein, wenn der Betreiber eines Bades eine regelmäßige Eigenüberwachung durchführt, wie sie ihm unter anderem entsprechend dem technischen Regelwerk vorgegeben wird. Hierbei sind besonders $\mathrm{zu}$ beachten die notwendigen täglichen Aufzeichnungen nach DIN 19643-1:201211 und die Richtlinie der Deutschen Gesellschaft für das Badewesen 60.07 „Instandhaltung technischer Anlagen in Bädern“.

Abweichungen von Untersuchungsumfang und Untersuchungsfrequenz nach DIN 19643:2012-11 sind mit dem Gesundheitsamt abzustimmen.

\subsection{1 Überwachung durch den Betreiber}

Bei der betriebseigenen Überwachung ist es u. a. notwendig, dass der Betreiber eines Bades täglich mehrmals von jedem Beckenwasser die Hygiene-Hilfsparameter freies Chlor, $\mathrm{pH}$-Wert und Redoxspannung sowie das gebundene Chlor bestimmt oder bestimmen lässt und die ermittelten Werte in das Betriebsbuch einträgt:

1. dreimal am Tag hinsichtlich des Gehaltes an freiem und gebundenem Chlor,

2. einmal am Tag hinsichtlich der Einhaltung des in $\bullet$ Tab. 2 festgelegten $\mathrm{pH}$-Wertbereiches. Die bei Betriebsbeginn ermittelten Werte sind mit den von der Mess- und Regelanlage angezeigten Werten zu vergleichen. Werden Abweichungen festgestellt, müssen die Geräte neu justiert oder instand gesetzt werden,

3. zweimal am Tag hinsichtlich der Nichtunterschreitung der in $\bullet$ Tab. 2 festgelegten minimalen Redoxspannung.

Die Werte der Redoxspannung werden von der Anzeige der Mess- und Regeleinrichtung übertragen. Bei Unterschreitung der unteren Werte nach $\bullet$ Tab. 2 um mehr als $50 \mathrm{mV}$ sind die Funktion und der Betrieb der Messeinrichtung und der Aufbereitungsanlage zu prüfen. Die Kontrolle und Justierung der Messeinrichtung für das Redoxpotenzial erfolgt nach Angaben des Herstellers.

\section{Erläuterung}

Die DIN 19643-1:2012-11 sieht in Kapitel 13.3 Absatz 2 ausdrücklich Handmessungen vor. Da gebundenes Chlor in der Regel nicht automatisch erfasst wird/werden kann, ist für die Bestimmung des gebundenen Chlors eine Handmessung erforderlich.

Die Redoxspannung kann nicht zuverlässig als Kurzzeitmessung von Hand bestimmt werden, da die Gleichgewichtseinstellung zwischen Wasser und Elektrode sehr lange dauern kann. Eine Kontrolle der Messeinrichtung erfolgt mit einem Standard-Redoxpuffer. In der Norm ist hierfür keine Frequenz vorgesehen.

Die im Rahmen der betriebseigenen Überwachung ermittelten Messdaten sind schriftlich oder auf Datenträgern in ein Betriebsbuch z. B. nach Abschn. 13.6.2 der DIN 19643-1:2012-11 einzutragen und durch alle weiteren Angaben zu ergänzen, die für eine betriebseigene Überwachung notwendig sind. Neben technischen Daten der Anlage sind dies z. B. Angaben zu Art und Menge der zugesetzten Aufbereitungschemikalien, Zahl der Badbesucher am Untersuchungstag oder durchgeführte Reinigungsarbeiten von Becken, Überlaufrinne und Wasserspeicher. Das Betriebsbuch sollte bis zu 10 Jahre aufbewahrt werden.

Im Rahmen der innerbetrieblichen Kontrolle ist es außerdem erforderlich, dass der Betreiber eines Bades folgende Untersuchungen (nach Tabellen 5 und 6 der DIN 19643-1:2012-11) ggf. in Absprache mit dem Gesundheitsamt durchführt 
oder durchführen lässt, wobei die Probennahme Betriebszustände mit Badebetrieb erfassen muss. Hierbei ist zu beachten, dass bei der aktuellen Norm DIN 19643-1:2012-11 die regelmäßige Untersuchung des Reinwassers durch die regelmäßige Untersuchung des Filtrats ersetzt wurde. Hierdurch soll frühzeitig eine erhöhte mikrobielle Belastung des Filters erkennbar sein, damit Maßnahmen eingeleitet werden können, bevor die Keimbelastung in Reinwasser und Beckenwasser durchschlägt:

1. mikrobiologische Untersuchungen zur Feststellung, ob die unter 2.1 festgesetzten Höchstwerte für die mikrobiologischen Parameter nicht überschritten sind

a) in Becken in geschlossenen Räumen und Becken, die sich zum Teil im Freien befinden, sowie in ausschließlich zu Saunabetrieben gehörenden Kaltwasserbecken im Freien im Abstand von längstens einem Monat,

b) in sonstigen Becken im Freien mindestens dreimal in der Badesaison, bei starker Nutzung mindestens zweimal monatlich,

c) im Füllwasser, wenn es nicht aus der öffentlichen Wasserversorgung stammt, in Abständen wie unter 2.3.1 1.a) bzw. 1.b) festgelegt. Eine Untersuchung auf Legionella species ist in der Regel nicht erforderlich,

d) im Filtrat wie unter 2.3.1 1.a) bzw. 1.b) festgelegt und

e) im Reinwasser bei Auffälligkeiten im Beckenwasser,

2. chemische Untersuchungen zur Feststellung, ob die in $\bullet$ Tab. 3 festgesetzten oberen Werte für die Parameter THM, Bromat sowie der Summenwert von Chlorit und Chlorat, die über den Zeitraum eines Jahres im Abstand von längstens 2 Monaten zu messen sind, nicht überschritten werden. Wenn die Parameterhöchstwerte in diesem Zeitraum nicht überschritten wurden, kann das Untersuchungsintervall auf längstens $4 \mathrm{Mo}$ nate ausgedehnt werden. Bei Anwendung von Chlor/Chlordioxid sollte die Untersuchung auf Chlorit im Ab- stand von längstens 2 Monaten erfolgen.

3. Untersuchung auf die sonstigen physikalisch-chemischen Parameter nach Tabellen 5 und 6 der DIN 19643-1:2012-11.

In Tabelle 5 der DIN 19643-1:2012-11 ist festgelegt, in welchen Bereichen des Wasserkreislaufs die jeweiligen Parameter - ggf. unter Einschränkung auf bestimmte Randbedingungen - zu untersuchen sind. Die Anforderungen für die Parameter im Filtrat sind für die verwendete Verfahrenskombination dem zugehörigen Folgeteil der DIN 19643:2012-11 in dem Abschnitt „Prüfung der Filtration“ zu entnehmen.

Wenn die chemische Beschaffenheit des Füllwassers starke Schwankungen aufweist, einer zusätzlichen Aufbereitung unterliegt oder eine Eigenversorgung vorhanden ist, ist es in die Untersuchung mit einzubeziehen. Dabei sind bei schwankender Zusammensetzung die Parameter Nitrat und Oxidierbarkeit zu untersuchen, damit die entsprechenden Werte aus den Becken bewertet werden können. Bei Füllwasser aus ungesicherten Vorkommen (kein Trinkwasser) sind zusätzlich die mikrobiologischen Parameter auf die Einhaltung der Anforderungen an Füllwasser (TrinkwV 2001 und zusätzlich $P$. aeruginosa nach DIN 19643:2012-11 ggf. bei Füllwasser $>23^{\circ} \mathrm{C}$ Legionella spp.) zu kontrollieren.

Die Untersuchungen einschließlich der Probennahmen sind nur durch ein akkreditiertes Labor nach den a. a. R. d. T. durchzuführen. Für die Probennahme zur Bestimmung der mikrobiologischen Parameter in $\bullet$ Tab. 1 gilt DIN EN ISO 19458. Zur Inaktivierung des freien Chlors ist eine Probennahmeflasche mit Natriumthiosulfat nach DIN EN ISO 19458 erforderlich.

Zur Bestimmung der chemischen und physikalisch-chemischen Parameter in den - Tab. 2 und 3 wird das Beckenwasser als Schöpfprobe aus dem oberflächennahen Bereich entnommen, etwa $10 \mathrm{~cm}$ bis $30 \mathrm{~cm}$ unter der Wasseroberfläche und etwa $50 \mathrm{~cm}$ vom Beckenrand entfernt.
Die Probennahmeflaschen können mit dem Probenwasser vorgespült werden, sofern keine Substanzen zur Probenstabilisierung wie Natriumthiosulfat vorgelegt sind.

Bei der Probennahme für die $\mathrm{pH}$ Wert-Kontrolle ist darauf zu achten, dass das zu untersuchende Wasser ohne Verlust an Kohlenstoffdioxid in die Probengefäße gelangt. Dazu wird das Wasser ohne Verwirbelung und Belüftung in das Probengefäß eingefüllt.

Proben zur Untersuchung auf leichtflüchtige Trihalogenmethane (THM) werden in Schliffstopfen-Flaschen genommen und müssen für die Zeit bis zur Untersuchung konserviert werden. Zur Reduktion des Restchlors, das während des Transportes und der Lagerung weiter unter THM-Bildung reagieren könnte, werden je $250 \mathrm{ml}$ Probe $20 \mathrm{mg}$ Natriumthiosulfat-Pentahydrat $\left(\mathrm{Na}_{2} \mathrm{~S}_{2} \mathrm{O}_{3} \cdot 5 \mathrm{H}_{2} \mathrm{O}\right)$ in die Probenflasche ge-

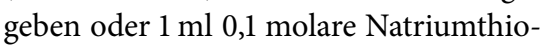
sulfat-Lösung vorgelegt. Zusätzlich werden zur Vermeidung der Nachbildung von THM ca. 200 mg Kaliumhydrogensulfat pro $250 \mathrm{ml}$ Probe zugesetzt. Bei Entnahme der Schöpfprobe wird die Flasche so lange untergetaucht, bis sie vollständig gefüllt ist. Die randvoll aufgefüllte Flasche wird sofort verschlossen, wobei das im Flaschenhals stehende Wasser durch den Schliffstopfen beim Verschließen verdrängt wird, sodass im Flaschenhals zwischen Wasserprobe und Schliffstopfen keine Luftblase entsteht.

Bei Horizontaldurchströmung des Beckens wird die Probe in der Mitte der Stirnseite des Beckens entnommen.

Bei Vertikaldurchströmung mit vollständigem Ablauf des Volumenstroms über die allseitig umlaufende Überlaufrinne sollte die Beckenwasserqualität an allen Stellen des Beckenrandes gleich sein. Die Probennahme kann beliebig längs des Beckenrandes erfolgen.

Für die Konservierung und Handhabung der Wasserproben gilt DIN EN ISO 5667-3.

Die Untersuchungen erfolgen nach den für die jeweiligen Parameter unter 2.1 und 2.2 vorgegebenen meist genormten Verfahren durch ein akkreditiertes Labor. Für den Fall der Überschreitung der mikrobiologischen Parameterhöchstwerte ist 
vertraglich sicherzustellen, dass das Labor den Betreiber sofort hierüber informiert. Der Betreiber muss das Gesundheitsamt unverzüglich über die Überschreitung der Parameterhöchstwerte unterrichten. Hierzu sollte eine unverzügliche Information des Gesundheitsamtes durch das Labor beauftragt werden.

Das Vorgehen bei Nichteinhaltung der Überwachungsparameter/Anforderungen ist in Abschn. 2.4 geregelt.

\subsection{2 Überwachung durch das Gesundheitsamt}

\subsubsection{Kontrollen}

Es ist notwendig, dass die Kontrollen durch das Gesundheitsamt bei der Erstinbetriebnahme eines Bades und dann grundsätzlich einmal im Jahr erfolgen. Geben die Kontrollen während eines Zeitraumes von 2 Jahren keinen Grund zu Beanstandungen, kann der Kontrollzeitraum auf einen 2-jährigen Rhythmus ausgedehnt werden. Die Kontrollen erfolgen im Rahmen einer Ortsbesichtigung einschließlich der Probennahme und beinhalten die Überprüfung

- der Erfüllung der Pflichten des Betreibers nach den Anforderungen der a. a. R. d. T. und nach den unter 2.3.1 aufgelisteten Empfehlungen,

- des Betriebsbuches auf richtige Führung und Auffälligkeiten,

- des Wasserkreislaufs des Beckenwassers einschließlich Wasseraufbereitung,

- der Trinkwasser-Installation einschließlich der Duschen sowie

- der hygienischen Anforderungen an sonstige Einrichtungen in Bädern.

Soweit die Probennahmen und Untersuchungen im Rahmen der Betreiberpflichten durch ein akkreditiertes Labor und in Abstimmung mit dem Gesundheitsamt durchgeführt wurden, kann dieses auf eine eigene zusätzliche Probennahme verzichten.

Als Arbeitshilfe für die Ortsbesichtigung durch das Gesundheitsamt wurde eine Checkliste erarbeitet (siehe http:// www.umweltbundesamt.de/themen/wasser/schwimmen-baden/schwimm-badebecken/empfehlungen-stellungnahmen). In schwierigen Fällen wird empfohlen, dass die Gesundheitsämter entsprechende Landeseinrichtungen (z. B. Hygieneinstitute oder Medizinaluntersuchungsämter/Landesgesundheitsämter) konsultieren.

\subsubsection{Untersuchungen}

Für die Untersuchungen von Wasserproben im Rahmen der amtlichen Überwachung gelten die Ausführungen unter 2.3.1 über deren Untersuchung einschließlich der Entnahme. Soweit die Untersuchungen der Wasserproben einschließlich der Probennahme nicht vom Gesundheitsamt selbst durchgeführt werden, haben sie ausschließlich durch eine für chemische und mikrobiologische Wasseruntersuchungen nach DIN EN ISO 17025 akkreditierte Untersuchungsstelle zu erfolgen. Die Überwachungsergebnisse sind mindestens 10 Jahre lang aufzubewahren.

\subsubsection{Kontrolle des Wasserkreislaufs}

Bei der Überwachung ist besonders auf folgende Bereiche bzw. Aspekte zu achten (hierbei handelt es sich um Stichwortangaben, Einzelheiten sind der DIN 19643:2012-11 zu entnehmen):

- Becken, Wasserspeicher und Filteranlagen,

- Dosierstellen für Chemikalien,

- Werkstoffe z. B. für Dichtungsmassen, Abdeckungen oder Auskleidungen,

- Einrichtungen mit zusätzlichen Wasserkreisläufen wie Rutschen, Wildwasserkanäle, Sprudler, Nackenduschen, Wasserpilze,

- das Wasser in den Zu- und Ableitungen und in den Pumpen (insbesondere auch nach Betriebsruhezeiten),

- Flächenreinigungsmittel für Beckenkopf mit Umgang,

- Wasserentnahme für den Betrieb von zusätzlichen Wasserkreisläufen (Reinwasser oder Beckenwasser) und

- Funktionskontrolle der Rinnenumschaltung.

\subsubsection{Trinkwasser-Installationen einschließlich Duschen}

Bei der Kontrolle der Trinkwasser-Installation (insbesondere der Duschen) durch das Gesundheitsamt gelten für Untersu- chungen und Maßnahmen die Vorgaben der TrinkwV 2001.

\subsubsection{Sonstige \\ Einrichtungen in Bädern}

Es ist unerlässlich, dass das Gesundheitsamt die Einhaltung der Anforderungen der Hygiene in sonstigen Einrichtungen des Bades in seine Überwachung einbezieht. Dies ist besonders wichtig z. B. für die Barfußbereiche, Sitzflächen, Sanitärbereiche, raumlufttechnische Anlagen und Attraktionen der Schwimmund Badebeckenanlage. Anhaltspunkte für die Überwachung liefern technische Regelwerke, wie z. B. die Richtlinie 94.04 „Hygiene, Reinigung und Desinfektion in Bädern" des Bundesverbandes Öffentliche Bäder e.V., die VDI-Richtlinien VDI 6022, VDI 6023 und VDI 2089 etc., sowie ggf. spezielle Empfehlungen des UBA und der BWK (z. B. die Empfehlung zur Lüftung in Hallenbädern) und der zuständigen Landesgesundheitsbehörden (s. auch Checkliste).

\subsection{Vorgehensweise und Maßnahmen bei Nichteinhaltung der Anforderungen}

Der Betreiber eines Bades ist dafür verantwortlich, dass eine Gesundheitsschädigung durch die Qualität des Schwimmund Badebeckenwassers für den Besucher nicht zu besorgen ist. Die Vorgehensweise bei Nichteinhaltung der mikrobiologischen und chemisch-physikalischen Anforderungen ist nachfolgend aufgeführt.

\subsubsection{Vorgehensweise und Maßnahmen des Betreibers bei mikrobiologischen Problemen}

Bei erhöhten mikrobiellen Werten im Filtrat sind Nachuntersuchungen in Filtrat und Beckenwasser durchzuführen. Bei erneut erhöhten Werten im Filtrat sollte insbesondere die Filterrückspülung auf ihre Effektivität überprüft werden. Solange die oberen Werte im Beckenwasser eingehalten werden, sind weitergehende Maßnahmen zum Schutz der Badegäste nicht erforderlich.

Bei Überschreitung der mikrobiologischen Parameterhöchstwerte unterrichtet das Untersuchungslabor im Falle von 


\begin{tabular}{|c|c|c|c|c|}
\hline \multirow{2}{*}{$\begin{array}{l}\text { Legionella } \\
\text { spp. } \\
\mathrm{KBE} / 100 \mathrm{ml}\end{array}$} & \multirow{2}{*}{$\begin{array}{l}\text { Bewer- } \\
\text { tung }\end{array}$} & \multicolumn{3}{|l|}{ Maßnahmen nach } \\
\hline & & Erstuntersuchung & $\begin{array}{l}\text { Nachuntersu- } \\
\text { chung }\end{array}$ & Weiteren Sanierungen \\
\hline$>1000^{a}$ & $\begin{array}{l}\text { Hohe Kon- } \\
\text { tamination }\end{array}$ & $\begin{array}{l}\text { Filterspülung, Des- } \\
\text { infektionsmittelzu- } \\
\text { gabe kontrollieren, } \\
\text { aerosolproduzieren- } \\
\text { de Einrichtungen } \\
\text { abschalten, Nach- } \\
\text { untersuchung, Kon- } \\
\text { trolle des Filtrats }\end{array}$ & $\begin{array}{l}\text { Nutzungsverbot, } \\
\text { Filterspülung, } \\
\text { Desinfektions- } \\
\text { mittelzugabe } \\
\text { kontrollieren, } \\
\text { Nachuntersu- } \\
\text { chung, Kontrolle } \\
\text { des Filtrats, Frei- } \\
\text { gabe nach ein- } \\
\text { wandfreiem mi- } \\
\text { krobiologischem } \\
\text { Befund }\end{array}$ & $\begin{array}{l}\text { Nutzungsverbot, weiter- } \\
\text { gehende Maßnahmen } \\
\text { unter Einbeziehung von } \\
\text { Fachleuten z. B. Hoch- } \\
\text { chlorung, Austausch des } \\
\text { Filtermaterials, wieder- } \\
\text { holte Nachuntersuchun- } \\
\text { gen von Beckenwasser } \\
\text { und Filtrat, Freigabe nach } \\
\text { einwandfreiem mikrobio- } \\
\text { logischem Befund }\end{array}$ \\
\hline $\begin{array}{l}>100 \text { bis } \\
1000\end{array}$ & $\begin{array}{l}\text { Mittlere } \\
\text { Kontami- } \\
\text { nation }\end{array}$ & $\begin{array}{l}\text { Filterspülung, Des- } \\
\text { infektionsmittelzu- } \\
\text { gabe kontrollieren, } \\
\text { Nachuntersuchung, } \\
\text { Kontrolle des Filtrats }\end{array}$ & $\begin{array}{l}\text { Filterspülung, } \\
\text { Desinfektions- } \\
\text { mittelzugabe } \\
\text { kontrollieren, } \\
\text { aerosolpro- } \\
\text { duzierende } \\
\text { Einrichtungen } \\
\text { abschalten, } \\
\text { Nachuntersu- } \\
\text { chung, Kontrolle } \\
\text { des Filtrats } \\
\end{array}$ & $\begin{array}{l}\text { Weitergehende Maßnah- } \\
\text { men unter Einbeziehung } \\
\text { von Fachleuten, z. B. } \\
\text { Hochchlorung, Austausch } \\
\text { des Filtermaterials, aero- } \\
\text { solproduzierende Ein- } \\
\text { richtungen abschalten, } \\
\text { wiederholte Nachunter- } \\
\text { suchungen von Becken- } \\
\text { wasser und Filtrat }\end{array}$ \\
\hline 1 bis 100 & $\begin{array}{l}\text { Geringe } \\
\text { Kontami- } \\
\text { nation }\end{array}$ & Nachuntersuchung & $\begin{array}{l}\text { Nachuntersu- } \\
\text { chung, Kontrolle } \\
\text { des Filtrats }\end{array}$ & $\begin{array}{l}\text { Nachuntersuchung, Kont- } \\
\text { rolle des Filtrats }\end{array}$ \\
\hline$<1$ & $\begin{array}{l}\text { Frei von } \\
\text { einer nach- } \\
\text { weisbaren } \\
\text { Kontami- } \\
\text { nation }\end{array}$ & Keine & - & - \\
\hline
\end{tabular}

aBei Legionellenkonzentrationen >10.000 KBE/100 ml und Legionellennachweis im Filtrat sofortige Nutzungsuntersagung.

Untersuchungen nach DIN 19643:2012-11 unverzüglich den Betreiber bzw. den Auftraggeber. Der Betreiber ist dafür verantwortlich, dass das Gesundheitsamt ebenfalls unverzüglich benachrichtigt wird. Der Betreiber ergreift erforderliche Maßnahmen und veranlasst ggf. eine sofortige weitere Untersuchung. Alle erforderlichen Maßnahmen und die weitere Vorgehensweise sollten zwischen Betreiber und Gesundheitsamt abgestimmt werden.

Die Überschreitung der einzelnen Parameterhöchstwerte birgt allerdings nicht identische Gesundheitsgefahren für die Benutzer des Bades.

Bei Nachweis von $P$. aeruginosa und E. coli ist eine höhere Gesundheitsgefahr als z. B. bei einer erhöhten Koloniezahl gegeben, da einerseits intestinale, direkt vom Menschen stammende Infektionserreger im Wasser vorhanden sein kön- ckenanlage verteilt wird), die Rinnenumschaltung vorzunehmen, das fäkale Material zu entfernen und soweit als möglich die Reste über die Rinne in den Schmutzwasserkanal zu spülen. Im Anschluss daran ist die Aufbereitungsanlage wieder in Betrieb zu nehmen und eine Hochchlorung (s. Anhang I) durchzuführen. Weiterhin muss eine mikrobiologische Kontrolluntersuchung veranlasst werden. Bei geringem Umfang der Verunreinigung kann das Becken am darauffolgenden Tag wieder freigegeben werden, wenn die Chlorwerte im zulässigen Bereich nach $\bullet$ Tab. 2 sind. In allen anderen Fällen ist wegen der höheren mikrobiologischen Belastung das Becken erst wieder freizugeben, wenn das Ergebnis der Kontrolluntersuchung vorliegt und der Höchstwert des entsprechenden Parameters (E. coli) nicht überschritten wird.

\section{Pseudomonas aeruginosa}

Pseudomonas aeruginosa kann durch Badegäste eingetragen werden und sich im Aufbereitungssystem vermehren. Neben dem Filtermaterial können auch wenigund nichtdurchflossene Rohrleitungen, fehlerhafte Innenauskleidungen, Pumpensysteme, Armaturen, Wärmetauscher, Dichtungsmaterial etc. besiedelt sein und zu einer Kontamination des Wassers führen. Das Beckenwasser und das Filtrat sollten gemäß DIN 19643:2012-11 parallel auf das Vorhandensein von P. aeruginosa untersucht werden, da nur dadurch eine differenzierte Aussage und Bewertung möglich sind. P. aeruginosa soll sowohl im Beckenwasser als auch im Filtrat in $100 \mathrm{ml}$ nicht nachweisbar sein. Ein gleichzeitig positiver Nachweis in beiden Wasserarten weist auf deutliche Probleme bei dem Bau oder dem Betrieb eines Bades hin und ist bedenklicher als ein alleiniger positiver Nachweis im Beckenwasser oder Filtrat einzuschätzen. Bei jedem positiven Nachweis ist sofort eine Nachkontrolle zu veranlassen und die Desinfektion bis zum Vorliegen eines negativen Nachweises engmaschig zu kontrollieren und im Rahmen der Vorgaben der DIN 19643:2012-11 der Gehalt an freiem Chlor mindestens im oberen Normbereich, maximal jedoch auf eine Konzentration von 1,2 mg/l einzustellen (s. Tab. 2). Ist P. aeruginosa in einer 


\begin{tabular}{|c|c|c|c|c|}
\hline \multirow{2}{*}{$\begin{array}{l}\text { Legionella } \\
\text { spp. } \\
\mathrm{KBE} / 100 \mathrm{ml}\end{array}$} & \multirow[t]{2}{*}{ Bewertung } & \multicolumn{3}{|c|}{ Maßnahmen nach } \\
\hline & & $\begin{array}{l}\text { Erstuntersu- } \\
\text { chung }\end{array}$ & $\begin{array}{l}\text { Nachuntersu- } \\
\text { chung }\end{array}$ & Weiteren Sanierungen \\
\hline$>1000$ & $\begin{array}{l}\text { Hohe Kon- } \\
\text { tamination }\end{array}$ & $\begin{array}{l}\text { Filterspülung, } \\
\text { Nachunter- } \\
\text { suchung des } \\
\text { Filtrats und des } \\
\text { Beckenwassers }\end{array}$ & $\begin{array}{l}\text { Filterspülung, } \\
\text { Überprüfung der } \\
\text { Aufbereitung, } \\
\text { Nachuntersuchung } \\
\text { des Filtrats und des } \\
\text { Beckenwassers, } \\
\text { ggf. Nutzungsein- } \\
\text { schränkung }\end{array}$ & $\begin{array}{l}\text { Weitergehende Maßnah- } \\
\text { men unter Einbeziehung } \\
\text { von Fachleuten, z. B. Hoch- } \\
\text { chlorung, Austausch des } \\
\text { Filtermaterials, Nachunter- } \\
\text { suchung des Filtrats und } \\
\text { des Beckenwassers, ggf. } \\
\text { Nutzungseinschränkung }\end{array}$ \\
\hline 1 bis 1000 & $\begin{array}{l}\text { Kontamina- } \\
\text { tion }\end{array}$ & $\begin{array}{l}\text { Filterspülung, } \\
\text { Nachunter- } \\
\text { suchung des } \\
\text { Filtrats und des } \\
\text { Beckenwassers }\end{array}$ & $\begin{array}{l}\text { Filterspülung, } \\
\text { Überprüfung der } \\
\text { Aufbereitung, } \\
\text { Nachuntersuchung } \\
\text { des Filtrats und des } \\
\text { Beckenwassers }\end{array}$ & $\begin{array}{l}\text { Weitergehende Maßnah- } \\
\text { men unter Einbeziehung } \\
\text { von Fachleuten, z. B. Hoch- } \\
\text { chlorung, Austausch des } \\
\text { Filtermaterials, } \\
\text { Nachuntersuchung des } \\
\text { Filtrats und des Becken- } \\
\text { wassers }\end{array}$ \\
\hline$<1$ & $\begin{array}{l}\text { Frei von } \\
\text { einer nach- } \\
\text { weisbaren } \\
\text { Kontamina- } \\
\text { tion }\end{array}$ & Keine & - & - \\
\hline
\end{tabular}

Konzentration $>10 \mathrm{KBE} / 100 \mathrm{ml}$ im Beckenwasser und/oder im Filtrat vorhanden oder sind wiederholt auch niedrigere P.-aeruginosa-Konzentrationen nachweisbar, sind die Filterspülung und Betriebsweise insgesamt zu überprüfen und ggf. zu optimieren sowie eine Hochchlorung des Beckenwassers bzw. der Anlagenteile (s. Anhang I) vorzunehmen. Je nach Situation vor Ort, z. B. bei einer bekannten Kontamination des Filtrats von $>10 \mathrm{KBE} / 100 \mathrm{ml}$ und im Falle einer zusätzlich auftretenden Störung der Chlordosierung oder der Regulation der Hygienehilfsparameter, kann auch eine Sperrung des Beckens notwendig sein. Welche Maßnahmen im Einzelfall angemessen, erforderlich und zielführend sind, hängt u. a. von der Höhe der gemessenen P.-aeruginosa-Konzentration, der Empfindlichkeit des betroffenen Personenkreises (z. B. Bäder in Krankenhäusern), den Parallel- oder Vorbefunden, die auf eine Kontamination (z. B. Biofilmbildung) in Anlagenteilen hindeuten und von Hinweisen auf Fehlfunktionen im Bereich der Steuerung der Chlordosierung und der Hygienehilfsparameter, Fehlfunktionen der Beckenhydraulik, des Rückspülprozesses etc. ab. Im Krankenhausbereich sind die erforderlichen Maßnahmen mit dem Krankenhaushy- gieniker abzustimmen. Die Desinfektion des Beckenwassers soll nicht dazu dienen, die Vermehrung von Krankheitserregern in den Aufbereitungsanlagen oder andere Unzulänglichkeiten des Betriebs auszugleichen oder zu kaschieren.

\section{Escherichia coli}

Wurde E. coli in einem Becken nachgewiesen, sind unverzüglich der Desinfektionsmittelgehalt und die Redoxspannung zu überprüfen, es ist umgehend die Ursache der Kontamination zu ermitteln und eine Nachprobe zu veranlassen. Nach Betriebsende (z. B. über Nacht) muss eine Hochchlorung des Beckenwassers durchgeführt werden. Sind nach diesen Maßnahmen wieder Chlorwerte gemäß - Tab. 2 und eine ausreichende Redoxspannung erreicht, kann das Becken weiter betrieben werden.

\section{Coliforme Bakterien}

Weist das Untersuchungslabor zwar keine E. coli, aber als Nebenbefund andere coliforme Bakterien nach, sollte es dies dem Betreiber des Bades mitteilen. Es sollte geprüft werden, ob die Aufbereitung ordnungsgemäß funktioniert.

\section{Koloniezahl bei $36^{\circ} \mathrm{C}$}

Wird lediglich die Koloniezahl überschritten, ist dies meist auf eine kurzfristig zu geringe Desinfektionswirkung zurückzuführen. Die Ursachen hierfür können vielfältig sein. Aussagen hierüber geben die Hygiene-Hilfsparameter. Diese sind unverzüglich zu kontrollieren und daraus abgeleitete Maßnahmen zu ergreifen. Auch eine Anpassung der Aufbereitungsmodalitäten an ggf. zu hohe Besucherfrequenzen kann erforderlich sein (z. B. durch Erhöhen der Umwälzleistung, des Füllwasserzusatzes usw.). Eine erhöhte Koloniezahl bei ausreichender Desinfektionsmittelkonzentration kann auch ein Hinweis auf eine Biofilmbildung im System z. B. im Filter sein.

\section{Legionella species}

Werden Legionella species direkt im Beckenwasser nachgewiesen, besteht der Verdacht, dass der Filter bewachsen ist und die Kontamination vom Filter ausgeht. Ein Problempunkt kann dabei die unzureichende Spülung sein. Die Bewertung der nachgewiesenen Legionellenkonzentrationen und die Empfehlung notwendiger Maßnahmen sind in - Tab. 4 aufgeführt. Eine Unterrichtung der Gesundheitsbehörde muss bei positivem Nachweis erfolgen.

Eine Filtratuntersuchung sollte in jedem Fall parallel zur Beckenwasseruntersuchung erfolgen, sofern die Beckenwassertemperatur $>23^{\circ} \mathrm{C}$ ist. Die Bewertung positiver Befunde und die Empfehlung notwendiger Maßnahmen sind in

\section{- Tab. 5 aufgeführt.}

Auch wenn nur im Filtrat Legionellen nachweisbar sind, muss die Aufbereitungsanlage überprüft werden. Bei komplexeren Systemen sollten Stufenuntersuchungen erfolgen, um die Kontaminationsquelle zu lokalisieren. Die erforderlichen Maßnahmen zur Sanierung des kontaminierten Systems sind von den technischen Gegebenheiten vor Ort abhängig. Nach Sanierung des Systems sind zunächst kurzfristige, dann Untersuchungen in größeren Zeitabständen notwendig, um die Nachhaltigkeit der getroffenen Maßnahmen zu kontrollieren. 


\subsubsection{Vorgehensweise und Maßnahmen des Betreibers bei Nichteinhaltung der chemischen und chemisch- physikalischen Anforderungen}

Werden die chemischen und chemischphysikalischen Parameter des Wassers gemäß Tabelle 2 der DIN 19643:201211 während des Badebetriebes nicht eingehalten, ist dies ein Hinweis auf Funktionsmängel der Anlage bzw. zu geringen Füllwasserzusatz mit evtl. nachteiligen Auswirkungen auf die mikrobiologische Qualität des Wassers. Es ist deshalb notwendig, Abweichungen zu begegnen, bevor die mikrobiologischen Höchstwerte überschritten werden.

Werden die 4 Überwachungsparameter freies Chlor, gebundenes Chlor, $\mathrm{pH}$ Wert und Redoxspannung eingehalten, kann in der Regel davon ausgegangen werden, dass die mikrobiologischen Parameterhöchstwerte nicht überschritten werden. Als erste Maßnahme muss deshalb versucht werden, mit der vorhandenen Technik die Einhaltung der $4 \mathrm{Hy}$ giene-Hilfsparameter zu erreichen. Kann dies mit den gegebenen technischen Anlagen nicht geleistet werden, sind schrittweise die Ursachen zu ermitteln und festgestellte Mängel zu beseitigen.

Zunächst ist festzustellen, ob die Flockungsmittelzugabe und das Spülprogramm der Filter richtig eingestellt sind.

Sind die Flockungsmittelzugabe und das Spülprogramm mangelhaft, dann führt dies zu einem Versagen der Flockungsfiltration mit Anstieg des gebundenen Chlors und Absinken der Redoxspannung als Folge, trotz ausreichenden freien Chlors und richtigen $\mathrm{pH}$-Wertes.

Wird damit kein ausreichender Erfolg erlangt/oder gewährleistet, dann sind die nachfolgend beschriebenen weitergehenden Untersuchungen mit Eingriffen in die Technik erforderlich:

- Die Säurekapazität des Beckenwassers ist zu überprüfen. Ist diese zu gering, dann ist die volle Funktion der Flockungsfiltration nicht mehr gegeben, sodass Maßnahmen zur Anhebung der Säurekapazität zu ergreifen sind (s. DIN 19643-1:2012-11, Kapitel 11.3).

- Die Zugabe des Flockungsmittels ist auf DIN-gerechte Ausführung zu überprüfen. Hierzu sind in der DIN 19643:2012-11 die Kapitel zu beachten, die sich mit der Flockung befassen. Dies gilt insbesondere für die richtige Anordnung der Dosierstelle und der Vermischung sowie für die Mindestzugabe an Flockungsmittel $\left(0,05 \mathrm{~g} / \mathrm{m}^{3}\right.$ als Aluminium für Aluminiumsalze und $0,1 \mathrm{~g} / \mathrm{m}^{3}$ als Eisen für Eisensalze).

- Das Filtermaterial ist auf ausreichende Schütthöhe und die richtige Lage beim Filtervorgang zu überprüfen. Bei falscher Ausführung des Überlauftrichters tritt Muldenbildung ein mit der Folge einer mangelhaften Filterwirkung. Gegebenenfalls ist der Überlauftrichter so nachzubessern, dass eine gleichmäßige Beaufschlagung des Filtermaterials mit Rohwasser gesichert ist, womit die Muldenbildung vermieden wird.

- Wird trotz Einhaltung der Hygiene-Hilfsparameter eine erhöhte mikrobielle Kontamination festgestellt, dann kann die Ursache (neben Aggregatbildung bzw. Vorliegen der Mikroorganismen in Biofilmen) darin liegen, dass als Filtermaterial Kornaktivkohle verwendet wird, die entgegen den Vorgaben der DIN 19643:2012-11 mit Rohwasser beaufschlagt wird, ohne vorherige Zugabe von Ozon.

- Es ist sicherzustellen, dass das Spülprogramm den Vorgaben der DIN 19643:2012-11 entspricht (programmgesteuerter unterbrechungsfreier zeitlicher Ablauf, Erreichen der notwendigen Filterbettausdehnung, gleichmäßige Beaufschlagung mit Spülwasser, rückstaufreier Ablauf des Spülabwassers). Dies gilt insbesondere für das Einhalten der Spülgeschwindigkeit. Ist diese geringer als die für das jeweilige Filtermaterial vorgegebene Spülgeschwindigkeit, dann erfolgt kein ausreichender Austrag von Mikroorganismen und Schmutzstoffen.

- Die vorgenannten Hinweise gelten für eine Filtration mit Schnellfiltern nach DIN 19605. Sind Anschwemmfilter nach DIN 19624 vorhanden, dann gilt sinngemäß die gleiche Vorgehensweise unter Be- achtung der Besonderheiten der Anschwemmfiltration, beschrieben in DIN 19643:2012-11 in Kapitel 4.4.3. Hierbei ist besonders darauf zu achten, dass benutztes Anschwemmmaterial, das bei der Spülung anfällt, zu verwerfen ist. Sind Membranfilter als Ultrafiltration im Einsatz, können Unverträglichkeiten durch Versagen der Membrane entstehen. Dies kann durch einen Integritätstest erkannt werden.

Bei weiter ausbleibendem Erfolg ist eine umfassende Untersuchung des gesamten Systems einschließlich der Beckenhydraulik erforderlich. Hierbei ist auch zu überprüfen, ob die Messwasserentnahmestelle im Becken so angeordnet ist, dass das entnommene Messwasser repräsentativ für das gesamte Beckenwasser ist.

\subsubsection{Vorgehensweise und Maßnahmen des Gesundheitsamtes}

Weist die Aufbereitung Mängel auf, entspricht das Wasser nicht den unter Abschn. 2.1 und 2.2 genannten Anforderungen oder sind Risiken für die menschliche Gesundheit absehbar, kann es notwendig sein, dass das Gesundheitsamt zusätzliche Untersuchungen veranlasst. Beispiele hierfür sind Untersuchungen an bestimmten Stellen des Wasserkreislaufes und zu bestimmten Zeiten, sofortige Untersuchungen außerhalb der regelmäBigen Untersuchungen, Untersuchungen häufiger oder in kürzeren Zeitabständen als unter 2.3.1 genannt, Untersuchungen auf andere Mikroorganismen bzw. chemische Stoffe als auf die in 2.1 bzw. 2.2 angeführten.

Wird dem Gesundheitsamt bekannt, dass die in 2.1 genannten Anforderungen an die mikrobiologischen Parameter nicht eingehalten werden oder Krankheitserreger bzw. chemische Stoffe im Beckenwasser enthalten sind, die eine Schädigung der menschlichen Gesundheit besorgen lassen, ist eine unverzügliche Prüfung erforderlich, ob das Wasser des betroffenen Beckens noch zum Schwimmen oder Baden zur Verfügung gestellt werden kann. Kommt der Betreiber seinen Pflichten nicht nach, sind in diesen Fällen darüber hinaus Abhilfe- 
maßnahmen anzuordnen oder Auflagen zu erteilen, um die Erfüllung der Anforderungen sicherzustellen. In Fällen, in denen die Anforderungen an die mikrobiologischen oder chemischen Parameter (- Tab. 1 und 2) nicht eingehalten werden bzw. die oben angegebenen zusätzlichen Untersuchungen hygienische Belastungen erkennen lassen, oder falls besondere Umstände hygienischer Beanstandungen es erfordern, ordnet das Gesundheitsamt entsprechend der Höhe des abschätzbaren gesundheitlichen Risikos ebenfalls Abhilfemaßnahmen an bzw. erteilt Auflagen.

Falls aufgrund der Überwachungsergebnisse oder aufgrund anderer Erkenntnisse eine Gesundheitsgefahr nicht auszuschließen ist, kann das Gesundheitsamt anordnen, dass das Wasser des betroffenen Beckens bis zur Behebung der Gefahrenlage nicht mehr zum Schwimmen oder Baden benutzt werden darf.

\section{Hygienische \\ Anforderungen an sonstige Einrichtungen in Bädern}

\subsection{Barfußbereiche, Sitzflächen und Sanitärbereiche}

Über Barfußbereiche, Sitzflächen und Sanitärbereiche kann es zur Übertragung von Krankheitserregern, wie z. B. Warzenviren, kommen. Aufgrund dessen ist es erforderlich, in Einrichtungen wie Schwimmbädern Hygienepläne zu erstellen. In diesen sollten die notwendigen Reinigungs- und Desinfektionsmaß nahmen detailliert beschrieben sein und den Beschäftigten als Arbeitsanweisung zur Verfügung stehen (s. auch Merkblatt 94.04 „Hygiene, Reinigung und Desinfektion in Bädern" der Deutschen Gesellschaft für das Badewesen).

Zur Vermeidung von Infektionen ist eine tägliche Reinigung und Desinfektion des Barfuß- und Sanitärbereiches sowie der Sitzflächen mit einem geeigneten Mittel [Liste des Verbundes für angewandte Hygiene (VAH) bzw. des RobertKoch-Institutes] in ausreichender Konzentration und Einwirkzeit erforderlich. Zur Überprüfung der Reinigungs- und Desinfektionsmaßnahmen können Abklatschuntersuchungen nach entspre- chender Einwirkzeit durchgeführt werden. Hierbei ist jedoch zu beachten, dass Untersuchungen während des Badebetriebes keine Aussage über die Qualität der durchgeführten Desinfektionsmaßnahmen erlauben.

Auf die Verwendung von Fußdesinfektionssprühanlagen kann verzichtet werden [s. Mitteilung der Badewasserkommission des Umweltbundesamtes „Hygienische Überwachung öffentlicher und gewerblicher Bäder durch die Gesundheitsämter (Amtsarzt), Anhang 2: Empfehlung zu Fußsprühanlagen in Schwimmbädern und Saunen " Bundesgesundheitsblatt 40, 11 (1997) 435-440].

\subsection{Raumlufttechnische Anlagen}

Von raumlufttechnischen Anlagen können bei falschem Betrieb oder nicht ausreichender Wartung Gesundheitsrisiken ausgehen. Um diesen zu begegnen, sind folgende Regelwerke zu beachten:

- DIN EN 12792 und DIN EN 13779,

- VDI-Richtlinie 6022 „Hygienische Anforderungen an raumlufttechnische Anlagen",

- VDI-Richtlinie 2089 „Technische Gebäudeausrüstung in Schwimmbädern, Blatt 1 Hallenbäder",

- Richtlinie 60.07 der Deutschen Gesellschaft für das Badewesen „Instandhaltung technischer Anlagen in Bädern“.

Bei Anlagen mit Umluftbetrieb ist darauf $\mathrm{zu}$ achten, dass immer ein ausreichender Außenluftmassenstrom gegeben ist, sodass die Konzentration der in der Hallenluft befindlichen Schadstoffe, insbesondere Stickstofftrichlorid, die geltenden Empfehlungen nicht überschreitet. Ist durch das Regelwerk ein konzentrationsunabhängiger Mindest-Außenluftmassenstrom festgelegt, dann sind die dafür notwendigen minimalen Klappenöffnungswinkel zu kennzeichnen, damit das Einhalten des minimalen Außenluftmassenstroms leicht überprüft werden kann.

\subsection{Trinkwasser-Installationen in Schwimmbädern}

Für die Planung, Errichtung, Änderung, Instandhaltung und den Betrieb von
Trinkwasser-Installationen in Schwimmbädern gelten die Anforderungen der TrinkwV 2001 in Verbindung mit den allgemein anerkannten Regeln der Technik.

Für den Betrieb der Duschen und der Waschbecken ist in jedem Fall Trinkwasser gemäß TrinkwV 2001 zu verwenden, in keinem Fall aufbereitetes Spülabwasser (Wasser aus der Filterspülung) oder aufbereitetes Beckenwasser. Für den Betrieb von Toilettenspülungen können zusätzlich Anlagen installiert werden, die für diesen Zweck Wasser, das nicht die Qualität von Trinkwasser besitzt, abgeben. Dabei sind neben den Anforderungen des technischen Regelwerkes an solche Anlagen auch die Bestimmungen der TrinkwV 2001 zu beachten. Grundsätzlich sind auch Trinkwasser-Installationen nach $₫ 13$ Absatz 4 TrinkwV 2001 gegenüber dem Gesundheitsamt anzeigepflichtig. Darüber hinaus bestehen nach $\$ 13$ Absatz 2 Nr. 5 TrinkwV 2001 grundsätzliche Anzeigepflichten, sofern die Trinkwasserbereitstellung im Rahmen einer öffentlichen Tätigkeit erfolgt, d. h. z. B. bei Neu- oder Wiederinbetriebnahme, bei Eigentums- und Nutzerwechsel oder bei bautechnischen Veränderungen mit Auswirkungen auf die Trinkwasserqualität. Nach $₫ 17$ Absatz 2 TrinkwV 2001 ist für die Verteilung von Wasser, das keine Trinkwasserqualität besitzt, ein separates Leitungssystem erforderlich - dieses muss dauerhaft als Nichttrinkwasserentnahmestelle und farblich unterschiedlich gekennzeichnet sein.

Seit der Zweiten Verordnung zur Änderung der Trinkwasserverordnung vom 5. Dezember 2012 (BGBl. I S. 2562) muss jeder Inhaber einer Trinkwasser-Installation, die eine Großanlage zur Erwärmung des Wassers umfasst, an mehreren repräsentativen Probennahmestellen die Wasserqualität regelmäßig auf das Vorhandensein von Legionella spp. überprüfen ( $\$ 14$ Absatz 4 TrinkwV 2001). Als Großanlage wird dabei das Vorhandensein eines Speicher-Trinkwassererwärmers oder zentralen Durchfluss-Trinkwassererwärmers mit einem Inhalt von mehr als 4001 oder einem Inhalt von mehr als 31 in mindestens einer Rohrleitung zwischen Abgang des Trinkwassererwärmers und der Entnahmestelle bezeichnet. Die Frequenz der Untersuchungen richtet 
sich dabei nach der Anlage 4 Teil II Buchstabe b TrinkwV 2001.

\section{Anforderungen an die Schwimm- und Badebeckenanlage bei Neu- und Umbau von Bädern}

\subsection{Vor dem Neubau oder Umbau eines Bades}

Um hygienische Mängel schon im Vorfeld des Betriebes von Bädern zu vermeiden, sollte beim Neubau von Bädern oder bei wesentlichen Änderungen an bestehenden Bädern bereits in der Planungsphase neben der Beteiligung der Baubehörde, Planer und Architekten auch eine Bewertung des Bauvorhabens unter hygienischen Gesichtspunkten durch das Gesundheitsamt durchgeführt werden. Sollte das Gesundheitsamt nicht über die dazu notwendigen personellen Ressourcen verfügen, muss ein hygienisches Fachgutachten erstellt werden. Von der Einhaltung der hygienischen Anforderungen ist regelmäßig auszugehen, wenn die Planung, Installation und der Betrieb der Schwimm- und Badebeckenanlagen vollständig den a. a. R. d. T. entsprechen (z. B. DIN 19643:2012-11). Daher hat der Betreiber des Bades die Planung eines Bades oder dessen Umbau dem Gesundheitsamt rechtzeitig anzuzeigen und alle für die hygienische Bewertung erforderlichen Unterlagen und Pläne vorzulegen. Dieses hat zu erfolgen für die erstmalige Inbetriebnahme des Bades oder eines Teiles davon sowie für die Vornahme baulicher oder betriebstechnischer Änderungen des Bades, soweit sie Auswirkungen auf die Beschaffenheit des Wassers in den Schwimm- oder Badebecken sowie der allgemeinen Hygiene haben können.

Es sollte in jedem Fall ein hygienischtechnisches Gutachten unter Berücksichtigung der Kriterien und Anforderung der a. a. R. d. T., insbesondere der DIN 19643:2012-11 für die Beckenwasseraufbereitung und des DVGW-Arbeitsblattes W 551 für die Trinkwasser-Installation in den Duschen, Sanitärräumen und im Saunabereich erstellt werden, wenn begründet von den a. a. R. d. T. abgewichen werden soll. In das Gutachten sind auch Anforderungen an die Montage und In- betriebnahme aufzunehmen, soweit diese Einfluss auf die Hygiene haben. Die erforderlichen Kosten sollten schon bei der Ausschreibung und der Angebotserstellung mit eingeplant werden. Die erforderliche Zustimmung des Gesundheitsamtes zur Planung wird unter Berücksichtigung dieses Gutachtens erteilt.

\subsection{Während der Bauphase oder des Umbaus}

Während der gesamten Bauausführung ist eine Bauleitung erforderlich, die die Einhaltung der besonderen hygienischen Anforderungen sicherstellt und dokumentiert. Insbesondere der hygienische Umgang mit dem angelieferten Material und während der Montage der später mit dem Beckenwasser in Berührung kommenden Anlagenteile und Oberflächen bedarf besonderer Sorgfalt, um eine primäre mikrobiologische Kontamination $\mathrm{zu}$ vermeiden. Auf eine ordnungsgemäße Filterbefüllung, Spülung und Erstdesinfektion nach der Montage ist hinzuweisen.

\subsection{Nach Fertigstellung der Baumaßnahme}

Nach dem Abschluss der Bauarbeiten und der Inbetriebnahme der Aufbereitungstechnik, aber noch vor der allgemeinen Eröffnung oder Wiedereröffnung des Bades sollte eine Abnahme durch einen Sachkundigen unter Einbeziehung:

- der Untersuchung der Beckenwasserqualität entsprechend der DIN 19643:2012-11,

- der Übereinstimmung der Wasseraufbereitungsanlage mit den a. a. R. d. T. bzw. den genehmigten Abweichungen davon (DIN-Normen, Richtlinien der Deutschen Gesellschaft für das Badewesen),

- der Wasserbeschaffenheit in der Trinkwasser-Installation (insbesondere Warmwassersysteme)

erfolgen. Weiterhin muss ein Hygieneplan nach 3.1 vorliegen, der sicherstellt, dass die Barfußbereiche, Sitzflächen und Sanitärbereiche ausreichend gereinigt und desinfiziert werden können. Aufgrund dieser Abnahme kann dann das
Gesundheitsamt die Freigabe des Bades für die Öffentlichkeit erteilen. Nach der Inbetriebnahme wird nach einer angemessenen Frist eine Funktionsprüfung nach der Richtlinie 65.04 „Funktionsprüfung von Anlagen zur Aufbereitung von Schwimm- und Badebeckenwasser nach DIN 19643: 1997-04“ empfohlen. Die Abnahme nach der Vergabe- und Vertragsordnung für Bauleistungen (VOB) ist kein Ersatz für diese Funktionsprüfung. Diese sollte nicht im Auftragsumfang der Firma liegen, die mit der Erstellung der Anlage beauftragt ist, sondern direkt durch Beauftragung des Bauherrn erfolgen (Bauherrenaufgabe).

Wird ein Bad ganz oder teilweise außer Betrieb genommen, so sollte das dem Gesundheitsamt innerhalb von $3 \mathrm{Ta}$ gen angezeigt werden.

\section{Literatur}

1. IfSG vom 20. Juli $2000, \mathrm{BGBI}$ I S. 1045

2. DIN 19643 1-4:2012-11. Aufbereitung von Schwimm- und Badebeckenwasser. Beuth, Berlin

3. Trinkwasserverordnung in der Fassung der Bekanntmachung vom 2. August 2013 (BGBL. I S. 2977), die durch Artikel 4 Absatz 22 des Gesetzes vom 7. August 2013 (BGBL. I S. 3154) geändert worden ist

4. Häntschel D, Sauermann G, Steinhart H et al (1998) Urea analysis of extracts from stratum corneum and the role of urea-supplemented cosmetics. J Cosmet Sci 49:155-163

5. Dygutsch P, Kramer M (2012) Chlorit und Chlorat. Ein neuer Summenparameter der DIN 19643 zur Überwachung von Schwimmbeckenwasser. In: AB Archiv des Badewesens 3:166-178 


\section{Anhang l: Hochchlorung}

(Dienstanweisung der Deutschen Gesellschaft für das Badewesen)

\section{Zusätzliche Desinfektions- maßnahmen durch Hochchlorung im Bereich der Badewasseraufbereitung}

\section{Veranlassung}

Aus unterschiedlichen Ursachen können im Wasser von Schwimm- und Badebeckenanlagen erhöhte mikrobielle Belastungen auftreten, erkennbar an einem deutlichen Überschreiten der vorgegebenen Parameterwerte für die mikrobiologischen Anforderungen in Tabelle $2 \mathrm{der}$ DIN 19643:2012-11 (Aufbereitung von Schwimm- und Badebeckenwasser). Ist eine Überschreitung länger andauernd, dann ist eine zusätzliche Desinfektionsmaßnahme erforderlich.

Die nachfolgende Dienstanweisung soll Hilfestellung geben bei der Durchführung der notwendigen zusätzlichen Desinfektionsmaßnahme.

\section{Definitionen im Sinne dieser Dienstanweisung}

\section{Desinfektion}

Abtötung bzw. Inaktivierung bestimmter Mikroorganismen durch oxidierende Desinfektionsmittel.

\section{Desinfektionsmittel}

Chemische Stoffe, die zur Desinfektion verwendet werden.

\section{Desinfektionskapazität}

Aufrechterhaltung einer Desinfektionsmittelkonzentration über einen bestimmten Zeitraum bzw. in einem bestimmten Versorgungsbereich.

\section{Hochchlorung}

Das Erreichen einer hohen Desinfektionskapazität unter Verwendung des Desinfektionsmittels Chlor.

\section{Desinfektionsmittel}

Für die Desinfektion des Wassers im Becken dürfen nur Desinfektionsmittel eingesetzt werden, die den Vorgaben der DIN 19643-1:2012-11, entsprechen.

Für die Desinfektion von Anlagenteilen der Badewasseraufbereitungstechnik ist zusätzlich Chlordioxid verwendbar.

\section{Technische Voraussetzungen}

Da die zusätzlichen Desinfektionsmaßnahmen hohe Chlorkonzentrationen erfordern, sind die technischen Voraussetzungen zu schaffen, dass diese während der Desinfektionsmaßnahme vorgehalten werden können. Bei Hallenbädern ist in der Regel eine Dosierleistung für die Zugabe von Chlor in den Aufbereitungskreislauf von $2 \mathrm{~g} \mathrm{Cl}_{2}$ je $\mathrm{m}^{3}$ Filtrat gegeben. Diese Dosierleistung reicht nicht aus, die notwendige Chlorkonzentration zu erreichen. Es kann notwendig werden zusätzlich Chlor zu beschaffen in Form von Calciumhypochlorit, Natriumhypochlorit oder Chlordioxid.

Da Chlor bei zunehmendem $\mathrm{pH}$-Wert im zu desinfizierenden Medium stark an Desinfektionswirkung verliert, muss sichergestellt werden, dass vor Durchführung der zusätzlichen Desinfektionsmaßnahme der $\mathrm{pH}$-Wert unter 7,5 eingestellt wird, wobei angestrebt werden sollte, den in der DIN 19643-1:2012-11 genannten unteren Wert von 6,5 zu erreichen. Wird zur Desinfektion Natriumhypochlorit verwendet, dann muss beachtet werden, dass durch Zugabe des Natriumhypochlorits der $\mathrm{pH}$-Wert erhöht wird.

Vor Durchführung der zusätzlichen Desinfektionsmaßnahme ist zu überprüfen, ob alle Anlagenteile, die mit Wasser mit erhöhter Chlorkonzentration in Berührung kommen, ausreichend korrosionsbeständig sind.

Ebenfalls vor Durchführung der vorgesehenen zusätzlichen Desinfektionsmaßnahme ist zu überprüfen, welche Anforderungen sich aus der zutreffenden Abwassersatzung bezüglich des bei der Desinfektionsmaßnahme anfallenden Abwassers ergeben. Gegebenenfalls sind notwendige Maßnahmen zu ergreifen, um das bei der Desinfektionsmaßnahme anfallende Abwasser schadlos in die Abwasserkanalisation einleiten zu können.

\section{Durchführung}

\subsection{Vorbemerkung}

Von jeder Maßnahme ist ein Protokoll zu fertigen mit folgendem Inhalt:

Grund der Maßnahme, Datum, Abfolge der Maßnahmen, Dauer der Einwirkzeit, Menge und Konzentration des verwendeten Desinfektionsmittels, Chlorkonzentration während der Einwirkzeit und zu Beginn des Badebetriebes. Hierbei ist zu beachten, dass die DPD-Methode ab einer Konzentration von $5 \mathrm{mg} / \mathrm{l}$ freies Chlor nicht mehr funktioniert und deswegen die Proben mit chlorfreiem Wasser verdünnt werden müssen.

Die Arbeitsschutzbestimmungen für die anzuwendenden Mittel sind zu beachten. Hier gilt insbesondere die Unfallverhütungsvorschrift des Bundesverbandes der Unfallkassen (GUV-VD 5).

\subsection{Beckenwasser}

Eine erhöhte mikrobielle Belastung im Beckenwasser ist in der Regel der Anlass zur Durchführung einer zusätzlichen Desinfektionsmaßnahme in Form einer Hochchlorung.

Vor Beginn der Hochchlorung sind die Filter zu spülen.

Die bei der Desinfektionsmaßnahme zu erreichende Desinfektionskapazität ist gekennzeichnet durch eine Chlorkonzentration von mindestens $10 \mathrm{mg} / \mathrm{l}$ über eine Dauer von mindestens $2 \mathrm{~h}$.

Filter, die mit Kornaktivkohle belegt sind, sollten, wenn möglich, umfahren werden. Bei einer Filteranlage mit einer Adsorptionsstufe mittels Pulveraktivkohle ist nach der Spülung die Zugabe von Pulveraktivkohle zu unterbinden. Die Pulveraktivkohle-Dosierung bleibt während des Hochchlorungsvorganges außer Betrieb.

Nach Durchführung der Hochchlorung ist vor Inbetriebnahme des Beckens die Chlorkonzentration einzustellen, die den Vorgaben der Tabelle 2 der DIN 19643-1:2012-11, Teil 1, entspricht, falls notwendig durch Verwendung von $\mathrm{Na}$ triumthiosulfat oder Wasserstoffperoxid zur Entchlorung. 
Hinweis: Zur Eliminierung von $1 \mathrm{~g} \mathrm{Cl}_{2}$ werden benötigt bei

1. $\mathrm{Na}_{2} \mathrm{~S}_{2} \mathrm{O}_{3}$ : CAS 7772-89-7 (Gehalt 98\%) - 0,57 g

2. $\mathrm{Na}_{2} \mathrm{~S}_{2} \mathrm{O}_{3} \cdot 5 \mathrm{H}_{2} \mathrm{O}$ : CAS 10102-17-7 (Gehalt 99\%) - 0,88 g

3. $\mathrm{H}_{2} \mathrm{O}_{2}: 30 \%(\mathrm{w} / \mathrm{w})$ CAS 77-84-1 $1,45 \mathrm{ml}$

4. $\mathrm{H}_{2} \mathrm{O}_{2}: 35$ wt $\%$ in $\mathrm{H}_{2} \mathrm{O}-1,21 \mathrm{ml}$

Zeigt die Hochchlorung des Beckenwassers keinen dauerhaften Erfolg, dann liegt die Ursache für die erhöhte mikrobielle Belastung in der Aufbereitungsanlage begründet, die Anlagenteile beinhaltet, die kontaminiert sind und Mikroorganismen abgeben. In diesem Fall sind Maßnahmen gegen diese kontaminierten Anlagenteile notwendig.

\subsection{Anlagenteile}

Unter Anlagenteilen im Sinne dieser Dienstanweisung werden die wasserberührten Anlagenelemente der Badewasseraufbereitungsanlage verstanden, ohne die Becken und die Wasserspeicher.

Anlagenteile, insbesondere die Filtermaterialien, sind häufig von Mikroorganismen in einer Kolonieform befallen, die einer Desinfektion schwerer zugänglich sind als einzelne Mikroorganismen. Deswegen muss die vorzuhaltende Desinfektionskapazität folgende Merkmale ausweisen:

Die Chlorkonzentration sollte mindestens $10 \mathrm{mg} / \mathrm{l}$ betragen bei einer Einwirkdauer von mindestens $2 \mathrm{~h}$. Bei der Verwendung von Chlordioxid ist die Mindestkonzentration von $1,0 \mathrm{mg} / \mathrm{l} \mathrm{ClO}_{2}$ zu beachten.

Ist Legionellenbefall gegeben, dann ist die Chlorkonzentration auf $50 \mathrm{mg} / \mathrm{l} \mathrm{zu}$ erhöhen mit einer Einwirkzeit von mindestens $12 \mathrm{~h}$ und die Konzentration an Chlordioxid auf $10 \mathrm{mg} / \mathrm{l}$ mit einer Einwirkzeit von mindestens $24 \mathrm{~h}$.

Am Ende der Einwirkzeit muss das Desinfektionsmittel noch nachweisbar sein.

Nach Durchführung der Desinfektion sind Filter mit Chlorzugabe zu spülen. War die Desinfektion als Folge von Legionellenbefall erforderlich, dann kann bei der Spülung Chlor in Form von Chlordioxid zweckmäßig sein.
Sind Filter mit Kornaktivkohle belegt, dann ist die Spülung mit gechlortem Wasser bei bereits eingetretener Verkeimung wegen des Chlorabbaus an der Materialoberfläche oft nur unzureichend wirksam, sodass ein Kohlewechsel erforderlich ist. Ein Versuch zur Hochchlorung kann unternommen werden, dann ist darauf zu achten, dass trotz der hohen Chlorzehrung im austretenden Schlammwasser noch eine deutliche Chlor-Restkonzentration vorhanden ist. Dies erfordert eine Chlorzugabe von $5-10 \mathrm{~g} / \mathrm{m}^{3}$ in das Spülwasser. Außerdem ist $\mathrm{zu}$ beachten, dass ein mehrfacher Hochchlorungsvorgang durchzuführen ist (mindestens 3-mal) mit jeweiligem Abstand von ca. 30 min und jeweiliger Ableitung des Erstfiltrates.

Vor Inbetriebnahme der Anlage ist das Gleiche zu beachten, wie unter Punkt 5.2 ausgeführt.

\section{Weiteres Vorgehen}

Zeigen die zuvor beschriebenen zusätzlichen Desinfektionsmaßnahmen keinen dauerhaften Erfolg, dann sind abschnittsweise Untersuchungen notwendig, um die Ursache für die andauernde Kontamination in Erfahrung zu bringen. Gegebenenfalls zeigen die abschnittsweisen Untersuchungen als Ergebnis die Notwendigkeit, Umrüstungen an der Anlage vorzunehmen.

Abschließend wird darauf hingewiesen, dass die zusätzliche Desinfektionsmaßnahme nicht verwendet werden darf, um anlagenbedingte Funktionsmängel zu überdecken. Wenn diese erkannt werden, sind sie zu beseitigen. 


\section{Bekanntmachungen - Amtliche Mitteilungen}

\begin{tabular}{|c|c|}
\hline Stammd & ecken \\
\hline Bezeichn & \\
\hline Straße: & PLZ, Ort: \\
\hline Ansprect & \\
\hline Name: & \\
\hline Telefon: & Mobil: \\
\hline Fax: & E-Mail-Adresse: \\
\hline Betriebsle & \\
\hline Telefon: & Mobil: \\
\hline Fax: & E-Mail-Adresse: \\
\hline Techniscr & \\
\hline Telefon: & Mobil: \\
\hline Fax: & E-Mail-Adresse: \\
\hline Betreiber & \\
\hline Name: & \\
\hline Straße: & PLZ, Ort: \\
\hline Telefon: & Mobil: \\
\hline Fax: & E-Mail-Adresse: \\
\hline
\end{tabular}

Angaben zur Nutzung des Bades Besucherzahl max. pro Tag

Babyschwimmen:

zusătzliche Einrichtungen:

sonstiges

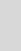

Art und Anzahl der Becken (gem. DIN 19643-1):

\begin{tabular}{|c|c|c|c|c|c|}
\hline & \begin{tabular}{|c|}
$\begin{array}{c}\text { Hallenbad } \\
(\mathrm{H})\end{array}$ \\
\end{tabular} & $\begin{array}{l}\text { Freibad } \\
(\mathrm{F})\end{array}$ & $\begin{array}{c}\text { Beckengröße } \\
\left(\mathrm{m}^{2}\right)\end{array}$ & \begin{tabular}{|c|}
$\begin{array}{c}\text { Beckenvolumen } \\
\left(\mathrm{m}^{3}\right)\end{array}$ \\
\end{tabular} & $\begin{array}{c}\text { Temperatur } \\
\left({ }^{\circ} \mathrm{C}\right)\end{array}$ \\
\hline \multicolumn{6}{|l|}{ Springerbecken } \\
\hline \multicolumn{6}{|l|}{ Schwimmerbecken } \\
\hline \multicolumn{6}{|l|}{ Variobecken } \\
\hline \multicolumn{6}{|l|}{ Wellenbecken } \\
\hline \multicolumn{6}{|l|}{ Nichtschwimmerbecken } \\
\hline \multicolumn{6}{|l|}{ Planschbecken } \\
\hline \multicolumn{6}{|l|}{ Wasserrutschenbecken } \\
\hline \multicolumn{6}{|l|}{ Kleinbecken } \\
\hline \multicolumn{6}{|l|}{ Bewegungsbecken } \\
\hline \multicolumn{6}{|l|}{ Therapiebecken } \\
\hline \multicolumn{6}{|l|}{ Durchschreitebecken } \\
\hline \multicolumn{6}{|l|}{ Warmsprudelbecken } \\
\hline \multicolumn{6}{|l|}{ Warmbecken } \\
\hline \multicolumn{6}{|l|}{ Kaltwassertauchbecken } \\
\hline \multicolumn{6}{|l|}{ Tretbecken } \\
\hline \multicolumn{6}{|l|}{$\begin{array}{l}\text { zusätzl. Wasserkreis- } \\
\text { lăufe od. Luftinjektionen }\end{array}$} \\
\hline & & & & & \\
\hline & & & & & \\
\hline & & & & & \\
\hline & & & & & \\
\hline & & & & & \\
\hline & & & & & \\
\hline
\end{tabular}

Bemerkungen 


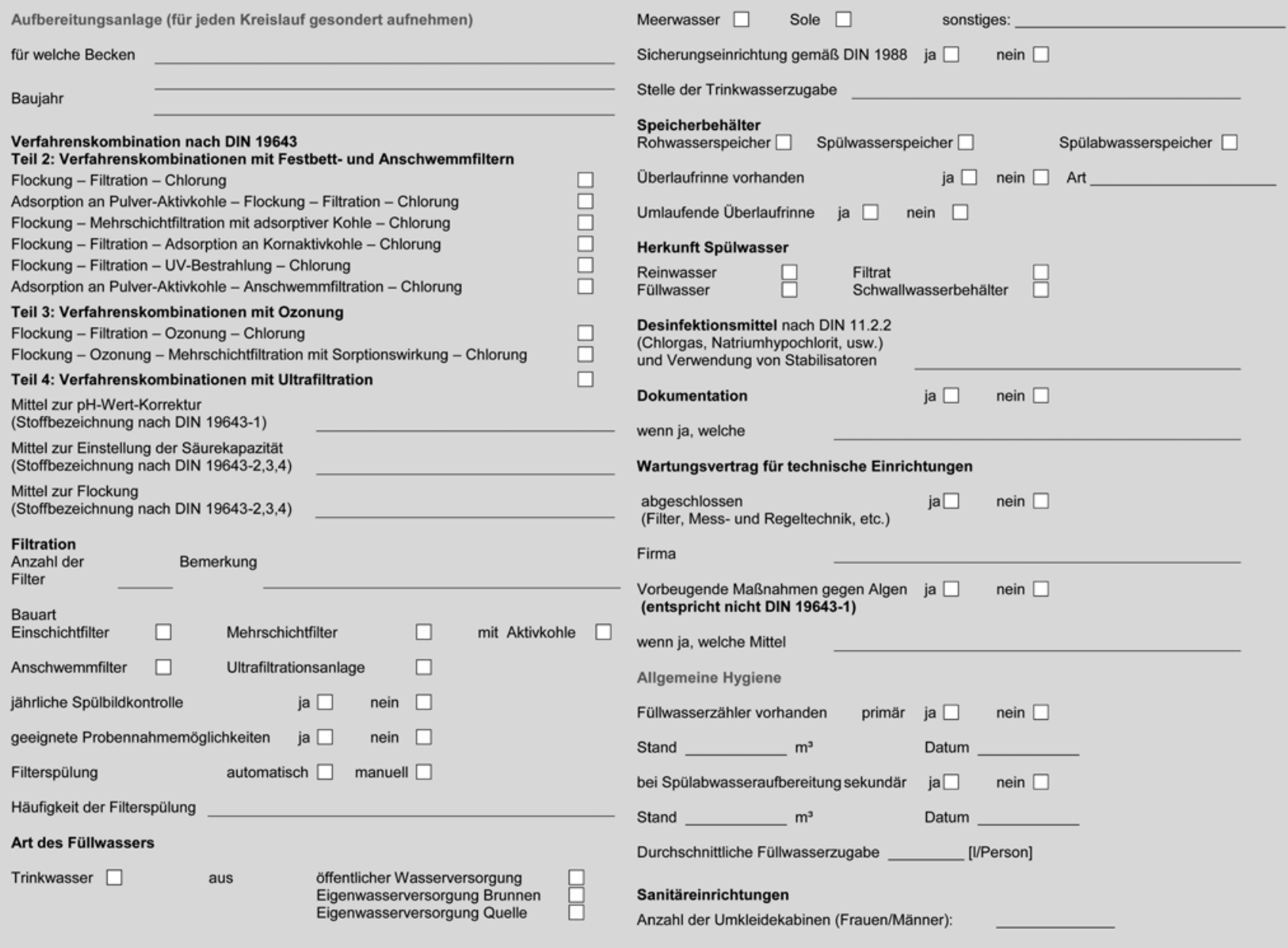

Abb. $1 \Delta$ Fortsetzung 


\section{Bekanntmachungen - Amtliche Mitteilungen}

\begin{tabular}{|c|c|c|c|}
\hline Anzahl der Duschen & Frauen & Mănner & \\
\hline Anzahl der Toiletten & Frauen & Mănner & \\
\hline barrierefreie Toilette & rhanden & ja $\square$ & nein \\
\hline barrierefreie Umkleic & vorhanden & ja $\square$ & nein \\
\hline Babywickelraum vorh & den & ja $\square$ & nein \\
\hline Raumlufttechnische & nlagen & & \\
\hline RLT-Anlage vorhand & & ja $\square$ & nein \\
\hline Betrieben nach VDI 2 & & ja $\square$ & nein \\
\hline Hygienekontrollen ge & VDI 6022: & ja $\square$ & nein \\
\hline $\begin{array}{l}\text { Automatische } \\
\text { Mess- und Regeltec }\end{array}$ & & ja $\square$ & nein \\
\hline
\end{tabular}

Beckendurchströmung (nach DIN 19643: 2012-11)

vertikal $\square$ horizontal $\square$

Der anschließende Teil des Fragebogens muss für jedes Becken gesondert ausgefüllt werden.

Allgemeine Angaben

Betriebsinterne Bezeichnung

Beckenart (nach Seite 2)

Ausführung

Baujahr

Sanierungen bzw. Umbaumaßnahmen

Becken-Volumenstrom

Aufbereitungs-Volumenstrom

Umwälzzeit

Nennbelastung (berechnet nach DIN 19643-1 Tab. 3)

Attraktionsplätze nach DIN Keramik $\square$ Kunststoff $\square$ Edelstahl $\square$

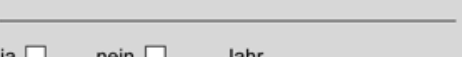

ja $\square \quad$ nein $\square \quad$ Jahr

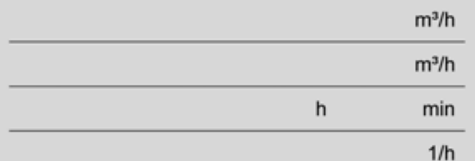

Bemerkungen

wenn ja, welche

nein $\square \quad$ ja $\square \quad$ Anzahl

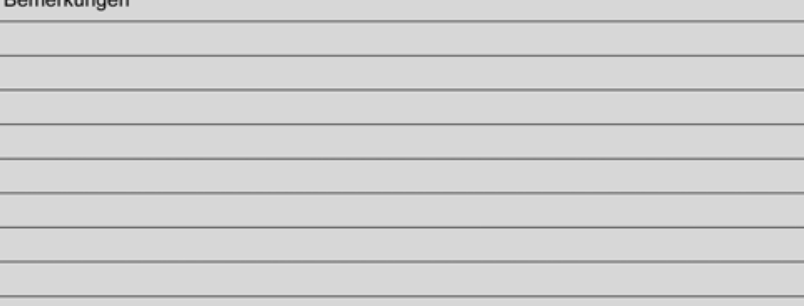

Abb. $1 \Delta$ Fortsetzung 


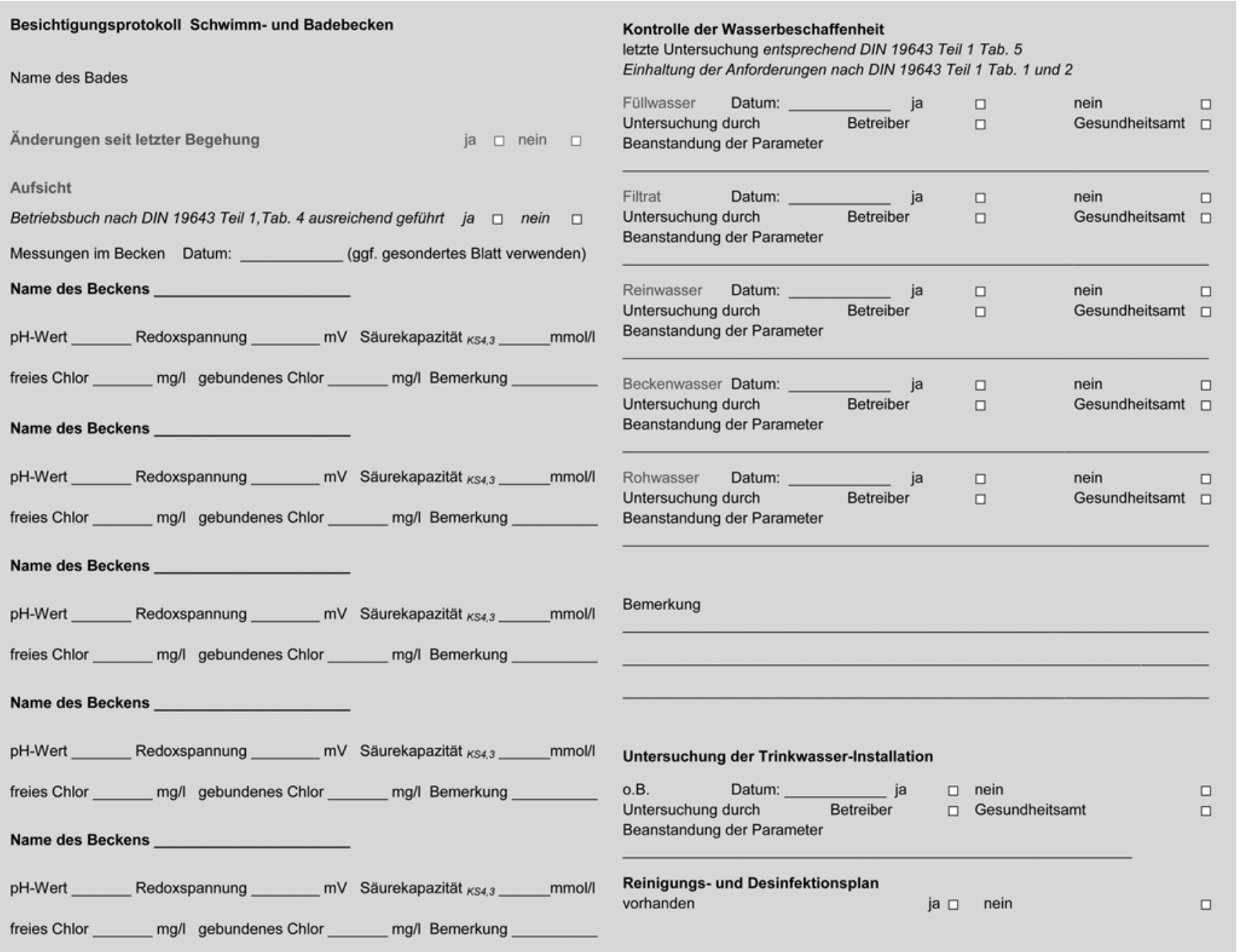

Abb. $1 \Delta$ Fortsetzung 


\section{Bekanntmachungen - Amtliche Mitteilungen}

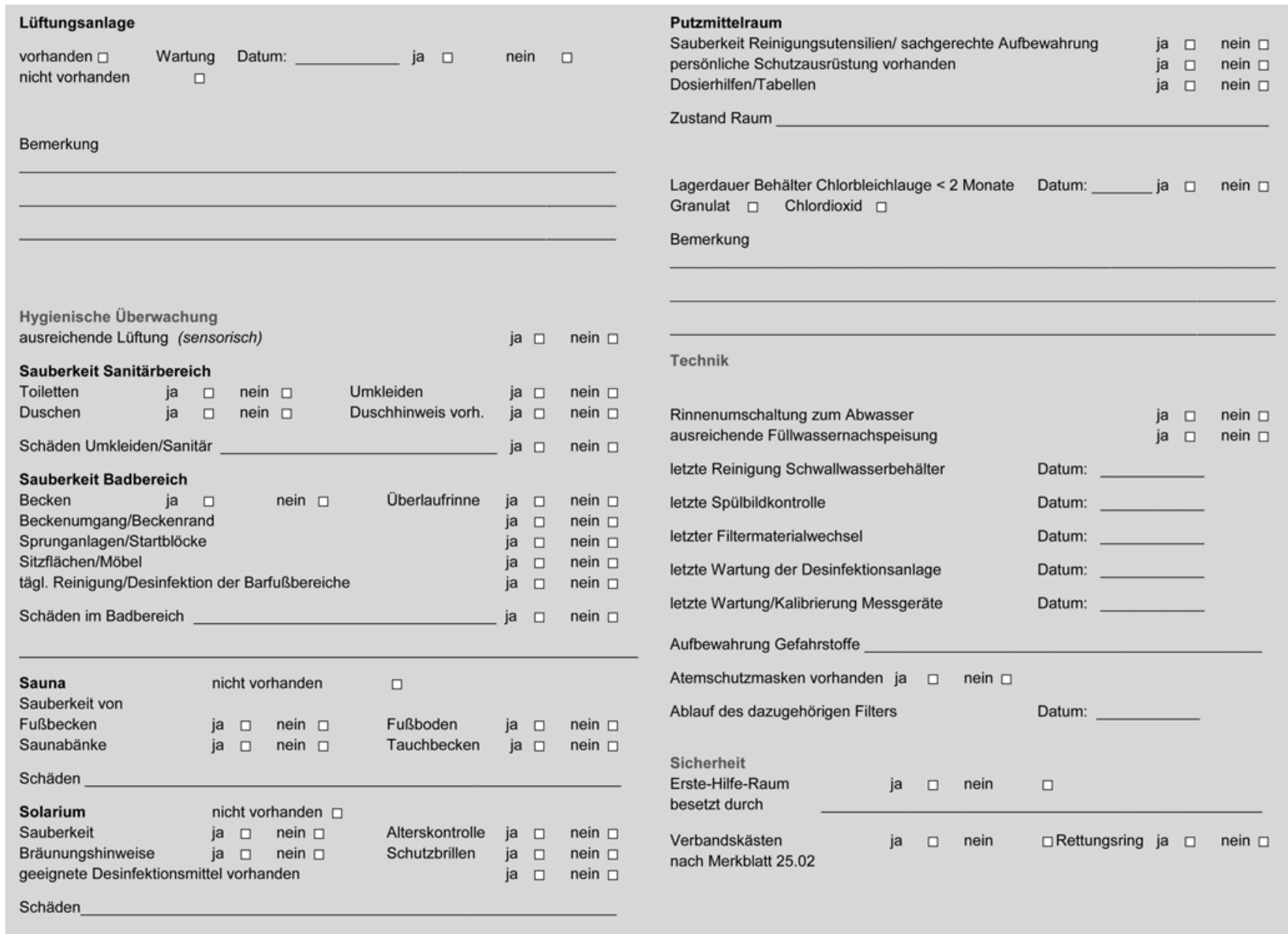

\section{Abb. $1 \Delta$ Fortsetzung}


Personalsituation

qualifizierte Fachkräfte vorhanden

geprüfte Meister/-innen für Badebetriebe und Schwimmmeister/-innen ja $\square \quad$ nein $\square$

Fachangestellte für Bäderbetriebe und Schwimmmeistergehilfen/-innen ja $\square$ nein

Bemerkung

$\overline{20.020}$

Maßnahmen

(20)

Datum: — Unterschrift:

Besichtigungsprotokoll Schwimm- und Badebecken (Bewertung)

Name des Bades

am:

durch:

Teilnehmer:

\section{Bemerkung:}

\section{Gesamtbewertung:}

das besichtigte Bad ist aus hygienischer Sicht einwandfrei $\square$ das besichtigte Bad weist aus hygienischer Sicht Mängel auf $\square$ Maßnahmen sind erforderlich

ja $\square$

nein $\square$

Abb. 1 A Fortsetzung 